OPEN ACCESS

Edited by: Claudio Bonghi,

University of Padua, Italy

Reviewed by:

Lijun Wang,

Insitute of Botany (CAS), China

Dong Meng,

Cornell University, USA

${ }^{*}$ Correspondence:

Li-Song Chen

lisongchen2002@hotmail.com

Specialty section:

This article was submitted to Crop Science and Horticulture,

a section of the journal

Frontiers in Plant Science

Received: 24 November 2016 Accepted: 24 February 2017

Published: 08 March 2017

Citation

Guo P, Qi Y-P, Yang L-T, Lai N-W, Ye $X$, Yang $Y$ and Chen L-S (2017)

Root Adaptive Responses to Aluminum-Treatment Revealed by RNA-Seq in Two Citrus Species With Different Aluminum-Tolerance.

Front. Plant Sci. 8:330 doi: 10.3389/fpls.2017.00330

\section{Root Adaptive Responses to Aluminum-Treatment Revealed by RNA-Seq in Two Citrus Species With Different Aluminum-Tolerance}

\author{
Peng Guo ${ }^{1}$, Yi-Ping Qi ${ }^{2}$, Lin-Tong Yang ${ }^{1}$, Ning-Wei Lai ${ }^{1}$, Xin Ye ${ }^{1}$, Yi Yang ${ }^{1}$ and \\ Li-Song Chen ${ }^{1,3,4 *}$
}

${ }^{1}$ Institute of Plant Nutritional Physiology and Molecular Biology, College of Resources and Environment, Fujian Agriculture and Forestry University, Fuzhou, China, ${ }^{2}$ Institute of Materia Medica, Fujian Academy of Medical Sciences, Fuzhou, China, ${ }^{3}$ Fujian Provincial Key Laboratory of Soil Environmental Health and Regulation, College of Resources and Environment, Fujian Agriculture and Forestry University, Fuzhou, China, ${ }^{4}$ The Higher Educational Key Laboratory of Fujian Province for Soil Ecosystem Health and Regulation, College of Resources and Environment, Fujian Agriculture and Forestry University, Fuzhou, China

Seedlings of aluminum (Al)-tolerant Citrus sinensis and Al-intolerant Citrus grandis were fertigated daily with nutrient solution containing 0 and $1.0 \mathrm{mM} \mathrm{AlCl}_{3} \bullet 6 \mathrm{H}_{2} \mathrm{O}$ for 18 weeks. The Al-induced decreases of biomass and root total soluble proteins only occurred in C. grandis, demonstrating that C. sinensis had higher Al-tolerance than C. grandis. Under Al-treatment, $C$. sinensis roots secreted more citrate and malate than $C$. grandis ones; less Al was accumulated in C. sinenis than in C. grandis leaves. The Al-induced reduction of phosphorus was lesser in $C$. sinensis roots and leaves than in $C$. grandis ones, whereas the Al-induced increase of sulfur was greater in C. sinensis roots and leaves. Using RNA-seq, we isolated 1905 and 2670 differentially expressed genes (DEGs) from Al-treated C. sinensis than C. grandis roots, respectively. Among these DEGs, only 649 DEGs were shared by the two species. Further analysis suggested that the following several aspects conferred $C$. sinensis higher Al-tolerance: (a) Al-treated C. sinensis seedlings had a higher external Al detoxification capacity via enhanced Al-induced secretion of organic acid anions, a higher antioxidant capacity and a more efficient chelation system in roots; (b) Al-treated $C$. sinensis seedlings displayed a higher level of sulfur in roots and leaves possibly due to increased uptake and decreased export of sulfur and a higher capacity to maintain the cellular phosphorus homeostasis by enhancing phosphorus acquisition and utilization; (c) Cell wall and cytoskeleton metabolism, energy and carbohydrate metabolism and signal transduction displayed higher adaptative responses to $\mathrm{Al}$ in $\mathrm{C}$. sinensis than in $\mathrm{C}$. grandis roots; (d) More upregulated than downregulated genes related to fatty acid and amino acid metabolisms were isolated from Al-treated $C$. sinensis roots, but the reverse was the case for Altreated C. grandis roots. These results provide a platform for further investigating the roles of genes possibly responsible for citrus Al-tolerance.

Keywords: aluminum, Citrus grandis, Citrus sinensis, organic acid anion secretion, phosphorus homeostasis, RNA-Seq, sulfur metabolism 


\section{INTRODUCTION}

In neutral or middly acidic soils, aluminum (Al) exists primarily as the forms of insoluble deposits. In acidic soils $(\mathrm{pH}<5.0)$, Al is released from these deposits into soil solution in the forms of $\mathrm{Al}^{3+}, \mathrm{Al}(\mathrm{OH})^{2+}$ and $\mathrm{Al}(\mathrm{OH})_{2}{ }^{+}$. Micromolar concentration of $\mathrm{Al}^{3+}$ is capable of inhibiting root growth, thus impairing water and nutrient uptake and leading to crop yield loss. Thus, Al-toxicity is a major limiting factor for crop productivity on acidic soils (Kochian, 1995; Yang et al., 2013). Citrus, one of the most important fruit crops in the world, are mainly cultivated on acidic and strong acidic soils. Low $\mathrm{pH}$ and high $\mathrm{Al}$ are the factors responsible for poor growth and shortened lifespan of citrus trees (Lin and Myhre, 1990). What's worse, soil acidification is occurring rapidly in citrus orchards in the last decade (Li et al., 2015). Therefore, understanding the mechanisms underlying Altoxicity and Al-tolerance in citrus plants is very important for citrus production.

To tolerate high level of active $\mathrm{Al}$ in acidic soils, higher plants have evolved diverse mechanisms for detoxifying externally such as the $\mathrm{Al}$-induced secretion of $\mathrm{Al}$ ligands [organic acid (OA) anions, phenolic compounds and phosphate $(\mathrm{Pi})]$ from the roots, efflux of $\mathrm{Al}$, increased $\mathrm{pH}$ in the rhizosphere, redistribution of $\mathrm{Al}$ and modified cell wall, and internally such as complexation and sequestration of Al. So far, the Al-induced release of OA anions from roots is the best characterized mechanism responsible for the Al-tolerance of higher plants (Yang et al., 2013; Kochian et al., 2015). Genes responsible for the Al-induced secretion of malate [ALMT1 (Al-activated malate transporter)] and citrate [HvMATE (multidrug and toxic compound extrusion) and SbMATE] have been cloned from wheat (ALMT1; Sasaki et al., 2004), barely (HvMATE; Furukawa et al., 2007) and sorghum (SbMATE; Magalhaes et al., 2007). Overexpression of these genes confers Al-tolerance in transgenic sorghum (Magalhaes et al., 2007), Arabidopsis (Liu et al., 2009), barely (Delhaize et al., 2004) and wheat (Pereira et al., 2010) plants.

Recently, several Al-tolerance genes involved in the cell wall modification [STAR1 (sensitive to Al rhizotoxicity1) and STAR2] and the sequestration of $\mathrm{Al}$ [ALS1 ( $\mathrm{Al}$ sensitive 1), ALS3 and Nrat1 (Nramp aluminum transporter 1) and IREG1 (IRON REGULATED/ferroportin 1)] have been identified in model plants rice (STAR1, STAR2 and Nrat1; Huang et al., 2009; Li et al., 2014), Arabidopsis (ALS1and ALS3; Larsen et al., 2005, 2007) and buckwheat (IREG1) (Yokosho et al., 2016). In addition, several Al-tolerance genes related to other processes that are independent of externall $\mathrm{Al}$ detoxification and sequestration of $\mathrm{Al}$ have been isolated from model plants, for example, OsART1 [Al resistance transcription factor (TF) 1 , a C2H2-type zinc-finger TF] (Yamaji et al., 2009), AtSTOP1 (sensitive to proton rhizotoxicity 1, encoding a zinc finger protein) (Iuchi et al., 2007), AtWRKY46 (Ding et al., 2013), OsASR5 (ABA stress and ripening, acting as a TF) (Arenhart et al., 2014), OsMGT1 [magnesium (Mg) transporter 1; Chen Z.C. et al., 2012], AtMGT1 (Deng et al., 2006) and OsMGD [monogalactosyldiacylglycerol (MGDG) synthase; Zhang et al., 2016]. All these genes have been demonstrated to confer Al-tolerance in transgenic rice, Arabidopsis and tobacco plants via overexpression and/or knockout (RNAi) of them.
Gene expression networks unraveled by transcriptomics give us the chance to understand the mechanisms of Al-toxicity and Al-tolerance in higher plants (Chandran et al., 2008; Kumari et al., 2008; Maron et al., 2008; Fan et al., 2014; Wang et al., 2015; Zhou et al., 2015). Recently, a highthroughput sequencing method [RNA sequencing (RNA-Seq)] is developed to analyze the transcriptome prior to the sequencing of the genome. It provides an opportunity for large-scale and simultaneous estimation of gene abundances and identification of new genes (Grabherr et al., 2011). RNA-seq has been applied to investigate Al-responsive genes in several higher plants including rice (Arenhart et al., 2014), Anthoxanthum odoratum (Gould et al., 2015), buckwheat (Fagopyrum tataricum) (Yokosho et al., 2014), Hydrangea macrophylla (Chen et al., 2015). Using the method, many candidate genes possibly responsible for $\mathrm{Al}$-tolerance have been identified in higher plants. However, most of these researches have focused on herbaceous plants and Alaccumulating plants. Limited data are available on Al-induced alterations of gene expression profiles in non-Al-accumulating woody plants (Brunner and Sperisen, 2013).

In China, citrus are cultivated commercially in acidic and strong acidic soils and are apt to suffer from high $\mathrm{Al}$ and low $\mathrm{pH}$ (Xu and Ji, 1998; Li et al., 2015). Previously, we used Al-tolerant Citrus sinensis and Al-intolerant Citrus grandis seedlings and comparatively investigated citrus Al-toxicity and Al-tolerance at physiological and protein levels (Yang L.T. et al., 2011; Jiang et al., 2015; Li et al., 2016). In addition, qRT-RCR analysis showed that the coordinated expression regulation of genes related to alternative glycolytic pathways, phosphorus (P) scavenging and recycling in C. sinensis and C. grandis roots played a role in citrus tolerance to $\mathrm{Al}$ and/or P-deficiency (Yang et al., 2012). In this study, we extended the knowledge on citrus Al-toxicity and $\mathrm{Al}$-tolerance through investigating the Al-induced alterations of transcriptomics in roots of the two citrus species with different Al-tolerance using RNA-Seq. Through analysis of the Al-responsive genes, we found some candidate genes possibly responsible for citrus Al-tolerance.

\section{MATERIALS AND METHODS}

\section{Plant Materials}

Seedling culture and $\mathrm{Al}$ treatments were carried out according to Zhou et al. (2015) with some modifications. Five-weeks after sprouting, uniform seedlings of 'Shatian pummelo' [Citrus grandis (L.) Osbeck] and 'Xuegan' [Citrus sinensis (L.) Osbeck] were transplanted to $6 \mathrm{~L}$ pots (two seedlings per pot) filled with clean river sand, then cultivated in a greenhouse with natural photoperiod at Fujian Agriculture and Forestry University throughout the trial period. Six weeks after transplanting, each pot was irrigated daily with nutrition solution containing $1 \mathrm{mM}$ $\mathrm{KNO}_{3}, 1 \mathrm{mM} \mathrm{Ca}\left(\mathrm{NO}_{3}\right)_{2}, 0.1 \mathrm{mM} \mathrm{KH} \mathrm{PO}_{4}, 0.5 \mathrm{mM} \mathrm{MgSO}_{4}$, $10 \mu \mathrm{M} \mathrm{H}_{3} \mathrm{BO}_{3}, 2 \mu \mathrm{M} \mathrm{MnCl}_{2}, 2 \mu \mathrm{M} \mathrm{ZnSO}_{4}, 0.5 \mu \mathrm{M} \mathrm{CuSO}_{4}$, $0.065 \mu \mathrm{M}\left(\mathrm{NH}_{4}\right)_{6} \mathrm{Mo}_{7} \mathrm{O}_{24}$ and $20 \mu \mathrm{M} \mathrm{Fe}$-EDTA, 0 (control, $-\mathrm{Al}$ ) or $1.0 \mathrm{mM}(+\mathrm{Al}) \mathrm{AlCl}_{3} \bullet 6 \mathrm{H}_{2} \mathrm{O}$ for 18 weeks until the sand was saturated. The $\mathrm{pH}$ of the solution was adjusted to $4.1-4.2$ with $\mathrm{HCl}$ or $\mathrm{NaOH}$. At the end of the experiment, approximately 
5-mm-long root tips from new white roots were excised, immediately frozen in liquid nitrogen, then stored at $-80^{\circ} \mathrm{C}$ until they were used for RNA and total soluble protein extraction. The remaining seedlings that were not sampled were used to measure biomass, root $\mathrm{OA}$ anion secretion, and root and leaf $\mathrm{Al}, \mathrm{P}$, and sulfur (S) concentrations.

\section{Seedling DW, S, Al and P in Root and Leaves, and Total Soluble Proteins in Roots}

At the end of this experiment, 10 seedlings per treatment (one seedling per pot) were collected and divided into roots, stems and leaves. Their DW was measured after being dried at $70^{\circ} \mathrm{C}$ for $48 \mathrm{~h}$. There were 10 replicates per treatment.

For the assays of S, Al and P in roots and leaves, fully expanded mature (about 7-week-old) leaves (midribs and petioles removed) and fibrous roots were collected after the seedlings were washed thoroughly with tap water and given a final rinsing with deionized water, then dried at $70^{\circ} \mathrm{C}$ for $48 \mathrm{~h}$. These dried samples were ground to pass a 40 -mesh sieve. $S$ was assayed with the vario MAX cube CNS analyzer (Elementar Analysensysteme GmbH, Hanau, Germany). Al and $\mathrm{P}$ concentrations were measured according to Hsu (1963) and Ames (1966), respectively after the powdered samples were digested in a mixture of $\mathrm{HNO}_{3}: \mathrm{HClO}_{4}$ (Yang et al., 2012). There were four replicates per treatment.

Root total soluble proteins were extracted with $50 \mathrm{mM}$ $\mathrm{Na}_{2} \mathrm{HPO}_{4}-\mathrm{KH}_{2} \mathrm{PO}_{4} \quad(\mathrm{pH} \quad 7.0)$ and $5 \% \quad(\mathrm{w} / \mathrm{v})$ insoluble polyvinylpyrrolidone. Total soluble proteins in the extract were assayed according to Bradford (1976). There were four replicates per treatment.

\section{Collection and Assay of Root Exudates}

Root exudates were collected according to Yang L.T. et al. (2011). At the end of this experiment, about 5-mm-long root apices from new white roots of $C$. sinensis and C. grandis seedlings treated with or without $1 \mathrm{mM}$ Al were excised. Ten-twelve excised root apices were collected in Petri dishes containing $5 \mathrm{~mL}$ of control solution (0.5 $\mathrm{mM} \mathrm{CaCl}_{2}, \mathrm{pH}$ 4.1-4.2). After three rinses with $5 \mathrm{~mL}$ of control solution (each for $20 \mathrm{~min}$ ), the root apices were transferred to $2 \mathrm{~mL}$ centrifuge tubes containing $1 \mathrm{~mL}$ of control solution in the presence or absence of $0.5 \mathrm{mM} \mathrm{AlCl}_{3} \bullet 6 \mathrm{H}_{2} \mathrm{O}(\mathrm{pH}$ 4.1-4.2). The tubes were placed vertically on a shaker (200 rpm) at dark. Citrate and malate secreted from roots were assayed according to Yang L.T. et al. (2011) as described above after 12 or $24 \mathrm{~h}$ treatment, respectively.

\section{RNA Extraction, cDNA Preparation and RNA-Seq}

Equal amounts of frozen roots from five seedlings (one seedling per pot) were pooled as one biological replicate. There were two biological replicates for each treatment. Total RNA was independently extracted twice from ca. 100 - $200 \mathrm{mg}$ frozen control and $+\mathrm{Al}$ roots of $C$. sinensis and $C$. grandis seedlings using Recalcirtant Plant Total RNA Extraction Kit (Centrifugal column type, Bioteke Corporation, China) following the manufacturer's instructions. The integrity and quality of total RNA were checked by $1 \%(\mathrm{w} / \mathrm{v})$ agarose gel electrophoresis and spectrophotometer at 260 and $280 \mathrm{~nm}$. Only these RNA samples that had a $260 \mathrm{~nm} / 280 \mathrm{~nm}$ absorbance ratio of between 1.8 and 2.0 were used for subsequent analyses. Ten microgram (500 ng/ $\mu \mathrm{L})$ of high quality total RNA per sample was shipped to the Genedenovo Biotechnology Corporation (Guangzhou, China) for deep sequencing and generation of datasets. Briefly, mRNA, which was isolated from approximately $5 \mu \mathrm{g}$ of total RNA, was enriched by using magnetic beads with oligo(dT) (Qiagen, Valencia, CA, USA). The resulting mRNA was then fragmented into short fragments (200 nt) and converted to the first-strand cDNA using random hexamer-primers. The second-strand cDNA was synthesized using RNase $H$ and DNA polymerase I, and purified using a QIAquick PCR extraction kit, then endrepaired with a base A tail adding to $3^{\prime}$ ends. Sequencing adapters were connected to the end of the double-stranded cDNA (200 $\pm 25 \mathrm{bp})$ for PCR amplification. Sequencing of the double-stranded cDNAs was carried out on $\mathrm{HiSeq}^{\mathrm{TM}} 2000$ device (Illumina Inc., San Diego, CA, USA) using the paired-end technology in a single run. Illumina GA Pipeline (version 1.6) was used to perform the original image process to sequences, basecalling and quality value calculation, in which $125 \mathrm{bp}$ paired-end reads were obtained (Li et al., 2013).

\section{RNA-Seq Reads Mapping, Transcript Assembly and Analysis of Gene Expression}

Clean reads were obtained by removing low quality sequences, reads containing adapter and reads containing ploy-N. In the mapping process, the software Bowtie aligner (Langmead et al., 2009) was used to delete the reads mapping to ribosomal RNA database. The remaining reads were mapped to the $C$. sinensis genome $^{1}$ using the software TopHat2 (Kim et al., 2013), and assembled by Cufflinks (Trapnell et al., 2010) and RABT (reference annotation based transcripts; Roberts et al., 2011).

The gene expression level was calculated by FPKM (fragments per kilobase of transcript per million mapped reads) method (Mortazavi et al., 2008). Differentially expressed analysis was performed using EdgeR (version 3.0.0, R version2.1.5). Genes that had a FDR (false discovery rate) $\leq 0.05$ and an absolute value of the $\log 2$ ratio $\geq 1$ were considered as differentially expressed. Their functional categories were assigned according to Uniprot ${ }^{2}$, $\mathrm{KEGG}^{3}$ and the gene ontology ${ }^{4}$ databases.

\section{qRT-PCR Analysis}

Root total RNA was extracted as described above. There were three biological replicates per treatment. Equal amounts of frozen roots from five seedlings (one seedling per pot) were mixed as one biological replicate. The replicate used for RNAseq and qRT-PCR was not the same. Genes specific primers were designed using Primier version 5.0 (Premier Biosoft International, Palo Alto, CA, USA). The sequences of the

\footnotetext{
${ }^{1}$ http://www.phytozome.net/search.php? method=Org_Csinensis

${ }^{2}$ http://www.uniprot.org/

${ }^{3}$ http://www.kegg.jp/

${ }^{4}$ http://www.geneontology.org/
} 


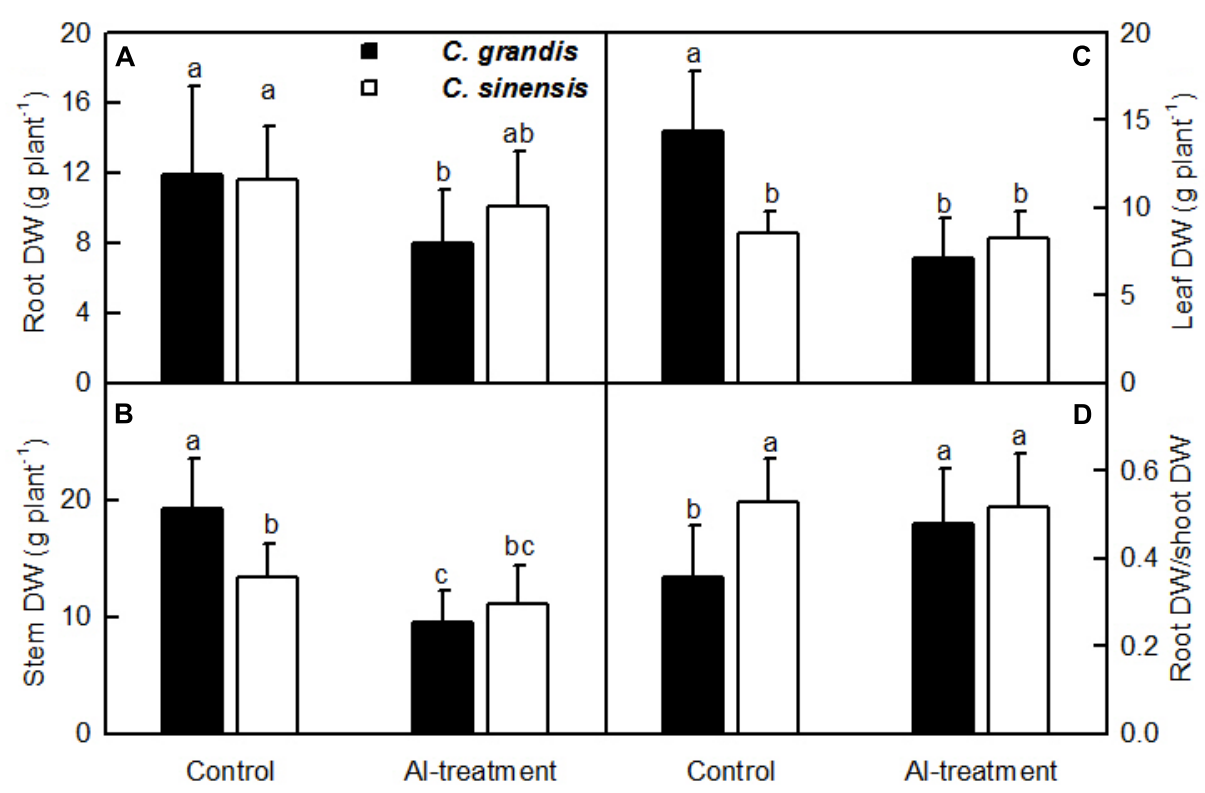

FIGURE 1 | Effects of Al-treatment on root (A), stem (B) and leaf (C) DW, and root DW/shoot DW ratio (D) of C. grandis and C. sinensis seedlings. Bars represent means $\pm \mathrm{SD}(n=10)$. Different letters above the bars indicate a significant difference at $P<0.05$.

Forward and Reverse primers were listed in Supplementary Table S1. qRT-PCR was carried out following the method of Zhou et al. (2013). For the normalization of gene expression and reliability of qRT-PCR data, three citrus genes [i.e., actin (JN191387), $\beta$-tublin (JN580571) and polyubiquitin (GU362416)] were selected as internal standards and the roots from -Al seedlings were used as reference sample, which was set to 1 . Each sample was run in two technical replicates.

\section{Experimental Design and Statistical Analysis}

There were 20 pots ( 40 seedlings) per treatment in a completely randomized design. Experiments were performed with 2-10 replicates. Results represented the mean $\pm \mathrm{SD}$. Differences among four treatment combinations (two Al levels $\times$ two species) were analyzed by ANOVA tests. Four means were separated by the Duncan's new multiple range test at $P<0.05$.

\section{RESULTS}

\section{Biomass, Al, $P$ and $S$ in Roots and Leaves, and Total Soluble Proteins in Roots}

Al-treatment reduced C. grandis root, stem and leaf DW. Root DW decreased to a lesser extent than shoot (stem + leaf) DW when exposed to $\mathrm{Al}$, thus leading to a greater ratio of root DW to shoot DW in C. grandis seedlings. However, Al-treatment did not significantly alter all the four parameters in $C$. sinensis seedlings (Figure 1).
$\mathrm{Al}$ concentration was higher in $C$. sinensis roots and leaves than in C. grandis roots and leaves in the absence of $\mathrm{Al}$, but was lower in Al-treated C. sinensis leaves than in Al-treated C. grandis leaves. No significant difference was found in Al level between $+\mathrm{Al}$ C. grandis and C. sinensis roots (Figures 2A,B). Al-treatment reduced $\mathrm{P}$ level in $C$. sinensis and C. grandis roots and leaves. Root and leaf $\mathrm{P}$ concentration did not differ between C. grandis and C. sinensis in the absence of $\mathrm{Al}$, but was lower in C. grandis than in C. sinensis under Al-treatment (Figures 2C,D). Al-treatment increased $\mathrm{S}$ level in C. sinensis roots and leaves and C. grandis roots, but did not significantly affect its level in C. grandis leaves. $S$ concentration was higher in C. sinensis roots and leaves than in C. grandis roots and leaves at each given $\mathrm{Al}$ level. The exception was that root $S$ level was similar between the two species at the absence of $\mathrm{Al}$ (Figures 2E,F).

Al-treatment lowered total soluble protein concentration only in C. grandis roots. Total soluble protein level was higher in C. grandis roots than in $C$. sinensis roots at the absence of Al, but the revers was the case under Al-treatment (Figure 2G).

\section{RNA-Seq, De novo Assembly of the Transcripts and Annotation}

Eight libraries were constructed and sequenced, including two biological replicates for control (control 1 and control 2) and Al-treated (Al-treatment 1 and Al-treatment 2) C. sinensis and C. grandis roots. The numbers of raw reads generated from each library ranged from $23,472,150$ to $29,474,312$. The percentages of clean reads and Q20 (sequencing error rates lower than 1\%) were more than 98 and 95\%, respectively (Table 1). Here, $75.2-83.8 \%$ of the clean reads were mapped uniquely to the $C$. sinensis genome, and only a small proportion of them were mapped multiply to the genome (Table 2). 


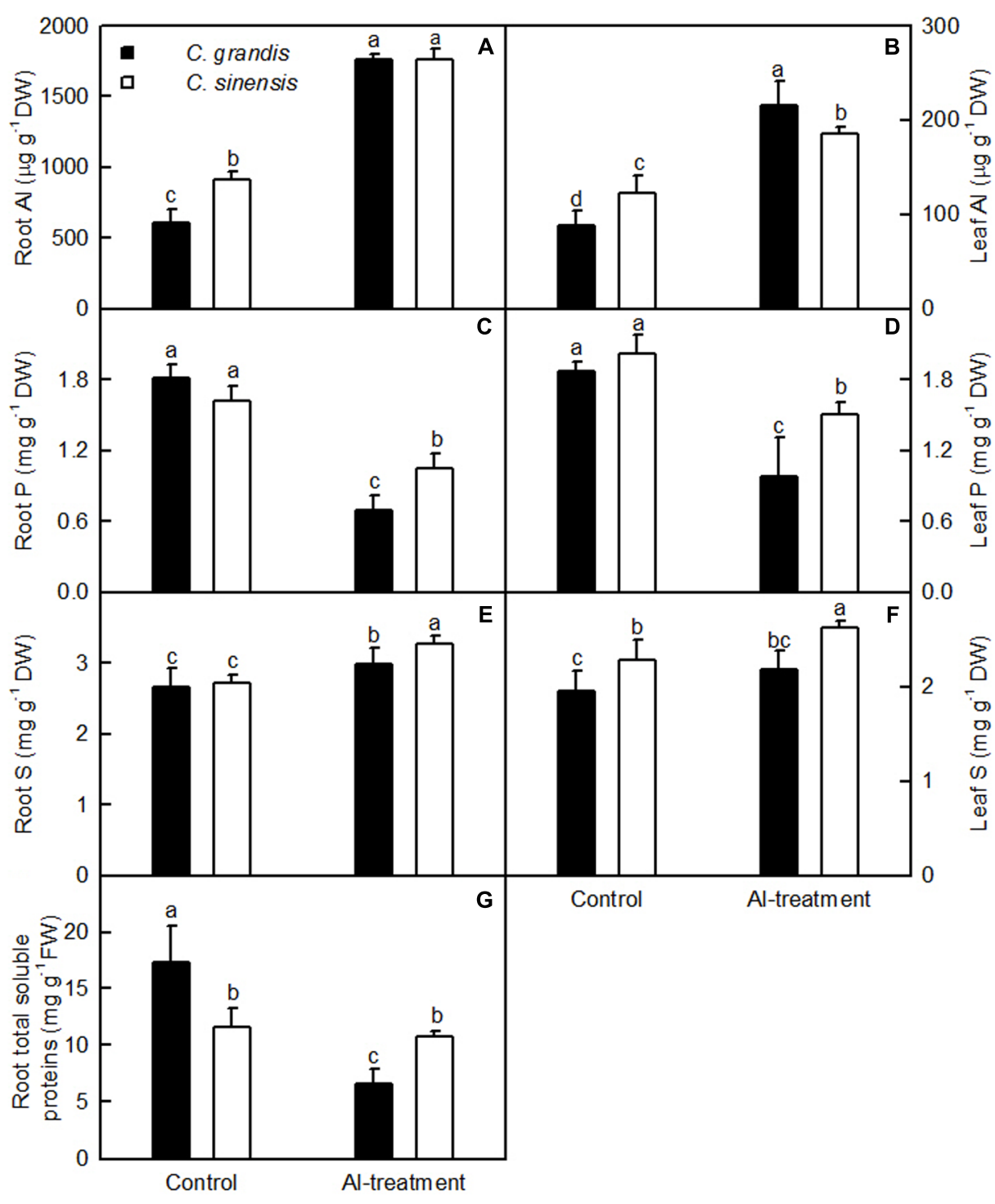

FIGURE 2 | Effects of Al-treatment on Al (A,B), P (C,D) and S (E,F) concentrations in C. sinensis and C. grandis roots (A,C,E) and leaves (B,D,F), and total soluble protein concentration in $C$. sinensis and C. grandis roots $\mathbf{( G )}$. Bars represent means $\pm \operatorname{SD}(n=4)$. Different letters above the bars indicate a significant difference at $P<0.05$.

Similar results have been obtained on C. grandis fruits (Guo et al., 2015). The number of known transcripts generated from reference genomes ranged from 20,846 to 21,953 , which accounted for $70.3-74.0 \%$ of the number of annotated genes in the genome. The number of known genes in the four groups (when mixed the two replicates to four groups: control C. sinensis, $+\mathrm{Al}$ C. sinensis, control C. grandis and $+\mathrm{Al}$ C. grandis) accounted for $73.8-76.3 \%$ of the annotated genes in the genome (Table 2).

\section{Al-Induced Secretion of Malate and Citrate}

The Al-induced secretion of malate and citrate from $+\mathrm{Al}$ excised roots was higher than from $-\mathrm{Al}$ excised ones. Excised C. sinensis roots secreted more citrate and malate than C. grandis ones when exposed to $\mathrm{Al}$ (Figure 3).

\section{DEGs between Control and Al-Treated Roots}

Here, the DEGs between control and Al-treated roots were identified with an absolute value of the $\log 2$ ratio $\geq 1$ and a threshold of FDR $\leq 0.05$. Based on the two criteria, we obtained 1293 upregulated and 1370 downregulated, and 990 upregulated and 915 downregulated DEGs from Al-treated C. grandis and C. sinensis roots, respectively (Figure 4A and Supplementary Tables S2, S3).

As shown in Figure 4B and Supplementary Table S4, we isolated a total of $3926 \mathrm{DEG}$ from $+\mathrm{Al}$ C. sinensis and C. grandis 
roots. Among these DEGs, only 649 DEGs were shared by the two species. The changes in low-P-responsive genes, TFs and genes involved in cellular transport, $\mathrm{S}$ transport and metabolism, antioxidation and detoxification, polysaccharide, cell wall and cytoskeleton metabolism, carbohydrate and energy metabolism, protein and amino acid metabolism, lipid metabolism, and signal transduction and hormone metabolism also differed between C. sinensis and C. grandis roots in response to $\mathrm{Al}$ (Supplementary Tables S5-14). Great differences existed in Al-induced alterations of gene expression profiles between the two citrus species. As shown in Figure 4C, 78.7\% of DEGs had an absolute value of $\log 2$ the ratio $<2$ in $+\mathrm{Al} C$. sinensis roots, while only $65.0 \%$ of DEGs had an absolute value of $\log 2$ the ratio $<2$ in $+\mathrm{Al}$ C. grandis ones, implying that wider variation of gene expression occurred in $+\mathrm{Al}$ C. grandis roots than in $+\mathrm{Al} C$. sinensis roots.

\section{qRT-PCR Validation of RNA-Seq Expression Data}

In order to validate RNA-Seq expression data, 60 DEGs selected randomly from C. grandis (30) and C. sinensis (30) roots were used for qRT-PCR analysis. Three genes were selected as the internal standards. The expression patterns of all 60 DEGs revealed by qRT-PCR were in agreement with the RNA-seq expression data, demonstrating that the RNA-seq data were reliable (Figure 5).

\section{DISCUSSION}

\section{Citrus sinensis Displayed Higher Al-Tolerance than C. grandis}

Al-treatment led to decreased root, stem and leaf DW, and increased ratio of root DW/shoot DW in C. grandis seedlings, but did not affect the four parameters in $C$. sinensis seedlings (Figure 1). Also, Al-induced reduction of the total soluble protein level only occurred in C. grandis roots (Figure 2G). Based on these results, we concluded that $C$. sinensis seedlings were more tolerant to $\mathrm{Al}$ than C. grandis ones. This is also supported by our data that less DEGs was identified in $+\mathrm{Al} C$. sinensis roots than in $+\mathrm{Al}$ C. grandis roots (Figure 4A). Similar result has been obtained on Al-treated maize roots (Maron et al., 2008). However, Jiang et al. (2015) obtained more Al-responsive proteins in C. sinensis than in C. grandis ones. The discrepancy between the Al-induced alterations of root gene expression patterns and protein profiles means that post-translational modifications (PTMs) might influence protein abundance.

The tolerance of higher plants to $\mathrm{Al}$ is related not only to low $\mathrm{Al}$ uptake, but also to relatively little $\mathrm{Al}$ transport from roots

TABLE 1 | Summary of the RNA-Seq data collected from control and Al-treated roots of C. sinensis and C. grandis.

\begin{tabular}{|c|c|c|c|c|c|c|c|c|c|}
\hline $\begin{array}{l}\text { Sample and } \\
\text { treatment }\end{array}$ & Raw reads & Clean reads (\%) & Read length & Adapter (\%) & Low quality (\%) & Poly A (\%) & $N(\%)$ & Q20 \% & GC \% \\
\hline \multicolumn{10}{|l|}{ C. sinensis } \\
\hline Control 2 & 28450848 & 28024122 (98.50\%) & $125+125$ & $194415(1.37 \%)$ & 18948 (0.13\%) & $0(0 \%)$ & $0(0 \%)$ & 95.47 & 45.02 \\
\hline Al-treatment 1 & 26476012 & 26081448 (98.51\%) & $125+125$ & 179110 (1.35\%) & 18172 (0.14\%) & $0(0 \%)$ & $0(0 \%)$ & 95.41 & 45.04 \\
\hline Al-treatment 2 & 24254284 & 23892372 (98.51\%) & $125+125$ & $164453(1.35 \%)$ & 16503 (0.14\%) & $0(0 \%)$ & $0(0 \%)$ & 95.43 & 45.14 \\
\hline Control 1 & 26043782 & 25665568 (98.55\%) & $125+125$ & $170586(1.31 \%)$ & 18521 (0.14\%) & $0(0 \%)$ & $0(0 \%)$ & 95.24 & 45.27 \\
\hline Control 2 & 26937382 & 26560774 (98.60\%) & $125+125$ & 170882 (1.27\%) & $17422(0.13 \%)$ & $0(0 \%)$ & $0(0 \%)$ & 95.59 & 45.52 \\
\hline Al-treatment 1 & 23472150 & 23203560 (98.86\%) & $125+125$ & $116640(0.99 \%)$ & 17655 (0.15\%) & $0(0 \%)$ & $0(0 \%)$ & 95.26 & 46.63 \\
\hline Al-treatment 2 & 28606284 & 28187340 (98.54\%) & $125+125$ & $192291(1.35 \%)$ & $17181(0.12 \%)$ & $0(0 \%)$ & $0(0 \%)$ & 95.69 & 44.46 \\
\hline
\end{tabular}

N, unknown base rates higher than 10\%; Q20, Sequencing error rates lower than 1\%.

TABLE 2 | Summary of clean reads and genes mapped to the reference genome from control and Al-treated roots of C. sinensis and C. grandis.

\begin{tabular}{|c|c|c|c|c|c|c|c|c|}
\hline $\begin{array}{l}\text { Sample and } \\
\text { treatment }\end{array}$ & Total reads & Total mapped & Multiple mapped & Unique mapped & Known genes & $\begin{array}{l}\text { Known genes in } \\
\text { two samples }\end{array}$ & $\begin{array}{l}\text { Novel } \\
\text { genes }\end{array}$ & $\begin{array}{l}\text { Total } \\
\text { genes }\end{array}$ \\
\hline \multicolumn{9}{|l|}{ C. sinensis } \\
\hline Control 2 & 28024122 & 23911090 (85.32\%) & 250294 (1.79\%) & 23410502 (83.54\%) & 21895 (73.8\%) & & 442 & 22337 \\
\hline Al-treatment 1 & 26081448 & 22299231 (85.50\%) & 231529 (1.78\%) & 21836173 (83.72\%) & $21953(74.0 \%)$ & $22629(76.3 \%)$ & 457 & 22410 \\
\hline Al-treatment 2 & 23892372 & 20453417 (85.61\%) & 212055 (1.78\%) & 20029307 (83.83\%) & 21789 (73.5\%) & & 454 & 22243 \\
\hline Control 1 & 25665568 & 19822398(77.23\%) & 193082 (1.50\%) & 19436234 (75.73\%) & $21171(71.4 \%)$ & $21871(73.8 \%)$ & 428 & 21599 \\
\hline Control 2 & 26560774 & 20540684 (77.33\%) & 219723 (1.65\%) & 20101238 (75.68\%) & 21078 (71.1\%) & & 418 & 21496 \\
\hline Al-treatment 1 & 23203560 & 18549555 (79.94\%) & 246157 (2.12\%) & 18057241 (77.82\%) & 20846 (70.3\%) & 21932(74.0\%) & 415 & 21261 \\
\hline Al-treatment 2 & 28187340 & 21615136 (76.68\%) & 208814 (1.48\%) & 21197508 (75.20\%) & 21377 (72.1\%) & & 425 & 21802 \\
\hline
\end{tabular}




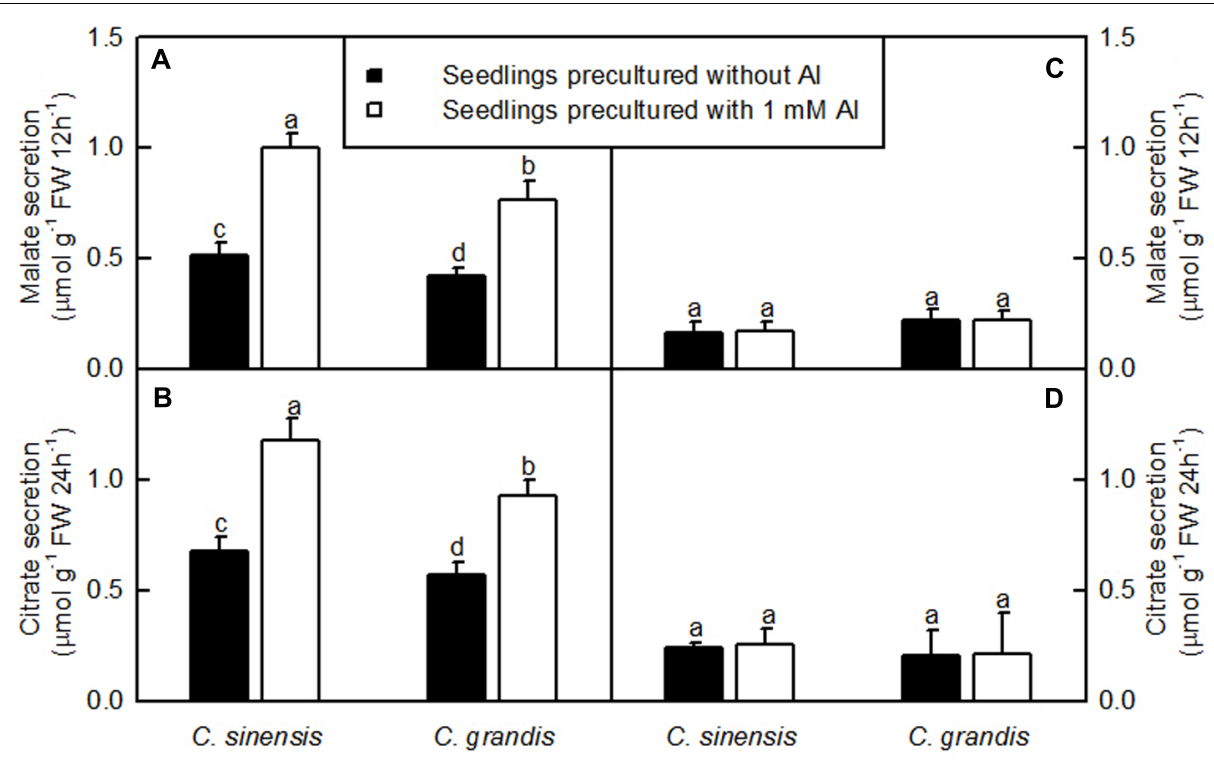

FIGURE 3 | Al-induced secretion of malate (A,C) and citrate (B,D) by excised roots from $\mathbf{C}$. sinensis and $\mathrm{C}$. grandis seedlings treated with 0 and $1 \mathrm{mM}$ Al for 18 weeks. Malate and citrate secretion from excised roots was determined after 12 or $24 \mathrm{~h}$ treatment, respectively in $0.5 \mathrm{mM} \mathrm{CaCl}_{2}+0.5 \mathrm{mM} \mathrm{AlCl}_{3} \bullet 6 \mathrm{H}_{2} \mathrm{O}(\mathbf{A}, \mathbf{B})$ or $0.5 \mathrm{mM} \mathrm{CaCl}_{2}(\mathbf{C}, \mathbf{D})$ solution, $\mathrm{pH}$ 4.1-4.2. Bars represent means $\pm \mathrm{SD}(n=4)$. Different letters above the bars indicate a significant difference at $P<0.05$.

to shoots (leaves) (Yang L.T. et al., 2011). Our results indicated that less Al was transported from roots to shoots (leaves) of C. sinensis seedlings when exposed to $\mathrm{Al}$ relative to C. grandis seedlings (Figures 2A,B), thus contributing to the Al-tolerance of $C$. sinensis seedlings.

The Al-induced secretion of $\mathrm{OA}$ anions from roots is considered as a major mechanism of Al-tolerance in higher plants (Yang et al., 2013; Kochian et al., 2015). We found that C. sinensis roots secreted more citrate and malate than $C$. grandis ones when exposed to $\mathrm{Al}$ (Figure 3), demonstrating that the Alinduced secretion of citrate and malate played a role in the higher Al-tolerance of $C$. sinensis via external detoxification. This is also supported by our finding that $\mathrm{Al}$ concentration was lower in $+\mathrm{Al}$ C. sinensis than in $+\mathrm{Al}$ C. grandis leaves (Figure 2B).

\section{Genes Related to Cellular Transport}

Many transport-related DEGs were isolated from $+\mathrm{Al}$ C. grandis and C. sinensis roots (Supplementary Table S5). They included genes encoding malate and citrate transporters [ALMTs, MATEs and ferric reductase defective $3 \mathrm{~b}$ (FRD3b)], ATPases, ion transporters, cyclic nucleotide-gated ion channels (CNGCs), ammonium transporters and amino acid transporters. Here, we obtained two upregulated and one downregulated, and two upregulated and three downregulated ALMTs from $+\mathrm{Al}$ C. grandis and C. sinensis roots, respectively, demonstrating the possible involvement of ALMTs in the Al-induced secretion of malate from citrus roots. However, the Al-induced secretion of malate from citrus roots could not be explained by the activation of ALMTs alone because C. sinensis roots secreted more malate than C. grandis roots when exposed to $\mathrm{Al}$ (Figures $3 \mathbf{A}, \mathbf{B}$ ). By contrast, we identified four upregulated genes (three MATEs and one FRD3b) involved in citrate secretion and one down-regulated
MATE from $+\mathrm{Al} C$. sinensis roots, but only one up-regulated $F R D 3 b$ and one down-regulated MATE from +Al C. grandis roots, which agrees with our report that the Al-induced secretion of citrate from C. sinensis roots was higher than from C. grandis roots (Figures 3C,D).

Internal detoxification of $\mathrm{Al}$ is mainly achieved via active transport and the sequestration of $\mathrm{Al}$ into the vacuoles (Kochian et al., 2015). Overexpression of FeIREG1 from buckwheat conferred Al-tolerance in transgenic Arabidopsis plants possibly through sequestrating $\mathrm{Al}$ into root vacuoles (Yokosho et al., 2016). Thus, the upregulation of Fe regulated 1 protein in $+\mathrm{Al}$ C. grandis and C. sinensis roots might be involved in citrus Al-tolerance. In rice, $\mathrm{Al}$ uptake is mediated by a plasma-membrane bound Nramp (natural resistance-associated macrophage protein) family Al transport protein, OsNarat1, which removes $\mathrm{Al}$ from the apoplast and sequester it into the vacuoles of roots by concerting with a vacuolar ABC-transporter, OsALS1 (Li et al., 2014). Here, we found that metal transporter Nramp6 was upregulated in $+\mathrm{Al}$ C. grandis roots. In addition, the expression of many genes encoding aquaporins, ABCtransporters and major facilitator superfamily proteins (MFSs) was altered in $+\mathrm{Al}$ C. grandis and C. sinensis roots, demonstrating the possible involvement of these genes in $\mathrm{Al}$ sequestration.

\section{Low-P-Responsive Genes}

Studies showed that P-deficiency was the key cause of the Al-induced growth inhibition in plants (Quartin et al., 2001), and that $\mathrm{P}$ supply could alleviate plant Al-toxicity (Jiang et al., 2009). Here, we obtained 16 upregulated and six downregulated, and 18 upregulated and three downregulated low-P-responsive genes from $+\mathrm{Al}$ C. grandis and C. sinensis roots, respectively, demonstrating that the upregulation of low-P-responsive genes 


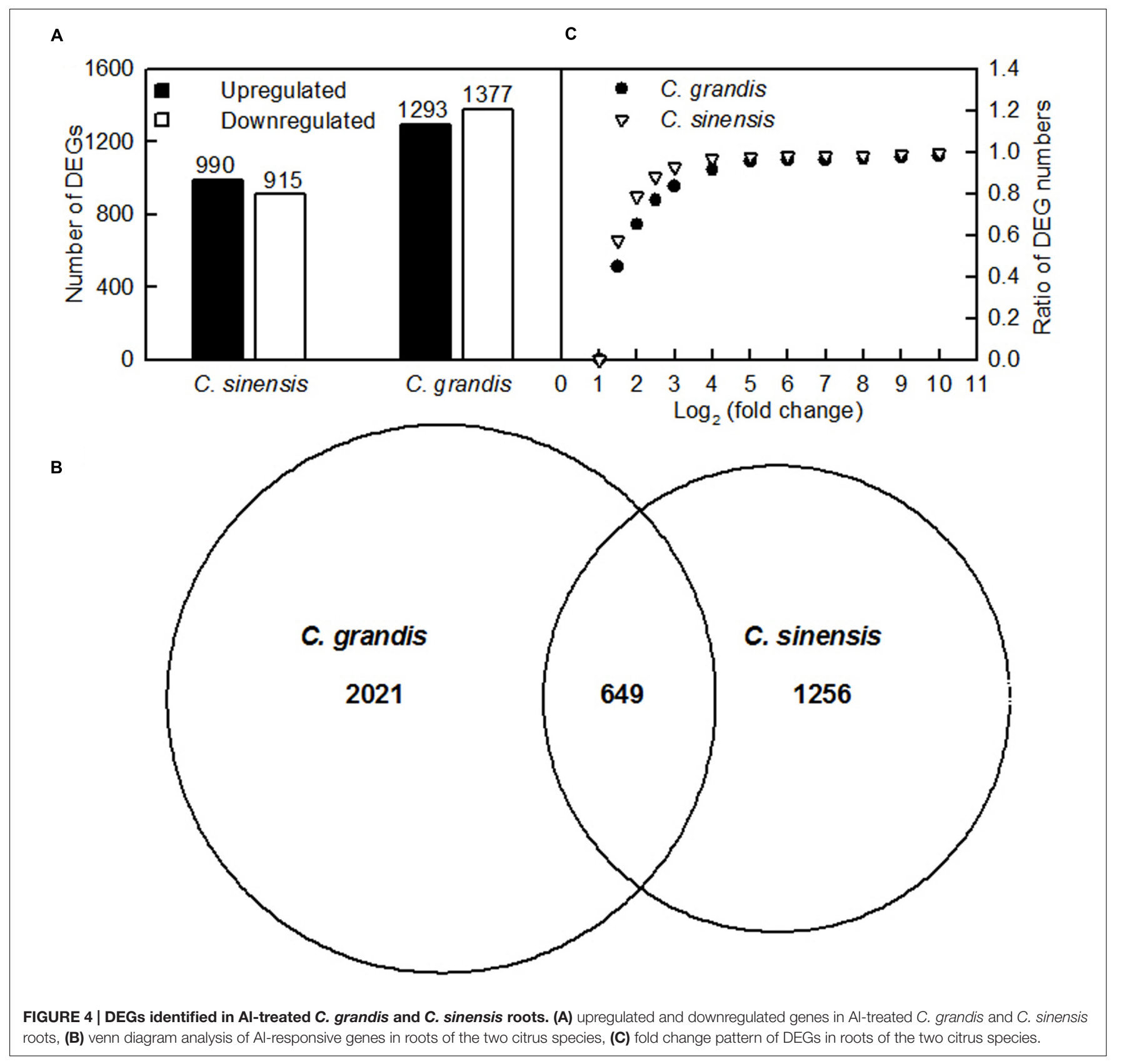

was greater in $+\mathrm{Al}$ C. sinensis roots (Supplementary Table S6). Induction of acid phosphatases (APs) is an adaptive stragety of plants to P-deficiency. Purple APs (PAPs, the largest group of non-specific APs) play a vital role in Pi recycling and scavenging in P-deficient plants (Liu et al., 2016). Maron et al. (2008) reported that the Al-induced upregulation of root PAPs was greater in Al-tolerant maize genotype than in Al-sensitive ones. Here, we isolated six upregulated and one downregulated PAPs from $+\mathrm{Al}$ C. sinensis roots, and one downregulated phosphatidic acid phosphatase-related protein, five upregulated and one downregulated PAPs from $+\mathrm{Al} C$. grandis roots. Also, we isolated one upregulated alkaline-phosphatase-like family protein isoform 1 from $+\mathrm{Al} C$. sinensis roots, which agrees with the report that an alkaline phosphatase was induced in P-deficient Phaseolus vulgaris roots (Morales et al., 2012). Duff et al. (1989) observed that NADP-glyceraldehyde-3-phosphate dehydrogenase (NADPG3PDH) specific activity was increased in P-starved Brassica nigra suspension cells, suggesting that the enzyme circumvented Pi-dependent NAD-G3PDH and phosphoglycerate kinase. Characterization of the Arabidopsis glycerophosphodiester phosphodiesterase (GDPD) family showed that plastid-localized AtGDPD1 played a role in maintaining cellular Pi homeostasis by releasing Pi from phospholipids in P-starved plants (Cheng et al., 2011). Ribonucleases play a role in the remobilization of Pi from RNA under P-limited conditions (Bariola et al., 1994). CaparrósMartín et al. (2013) showed that that AtSgpp (At2g38740) gene 
A

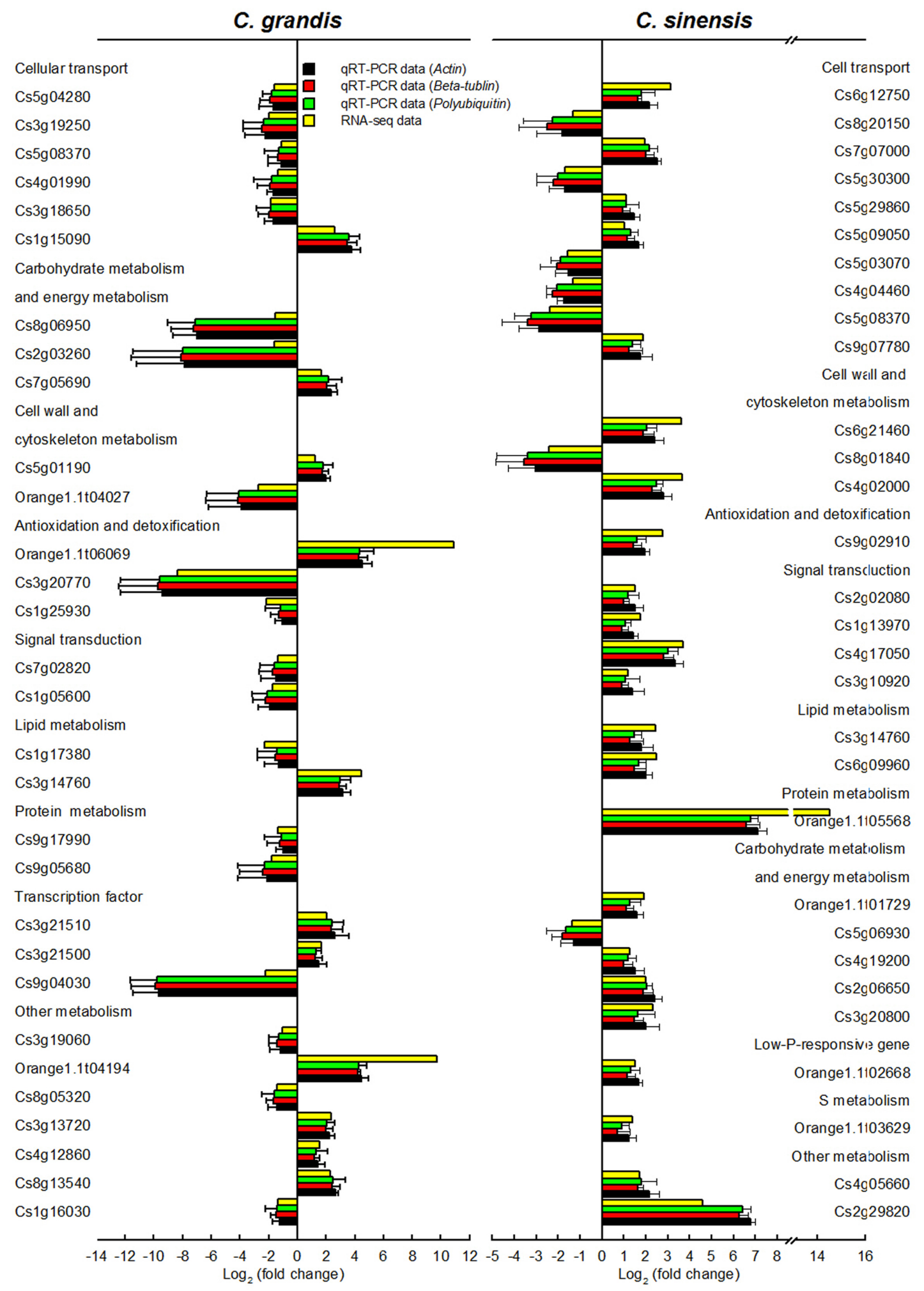

FIGURE 5 | Relative expression levels of DEGs from Al-treated C. grandis (A) and C. sinensis (B) roots. Bars represent means $\pm \mathrm{SD}(n=3)$. Three citrus genes [i.e., $\beta$-tubulin (JN580571), actin (JN191387) and polyubiquitin (GU362416)] were selected as the internal standards and the roots from control seedlings were used as reference sample, which was set to 1 . 
encoding haloacid dehalogenase-like hydrolase (HAD) might play a role in the homeostatic balance of $\mathrm{Pi}$ in the cell. Here, we isolated three and one upregulated HAD genes from $+\mathrm{Al}$ C. sinensis and C. grandis roots, respectively. Also, we obtained two downregulated (Cs2g04480 and Cs9g18560) and two upregulated (Cs3g27660 and Cs7g19830) genes involved in $\mathrm{Pi}$ transport from $+\mathrm{Al}$ C. grandis roots, but only one downregulated (Cs9g18560) and two upregulated (Cs7g19830 and Cs5g29860) genes from $+\mathrm{Al} C$. sinensis roots, indicating that Al-treatment affected Pi transport more in C. grandis than in C. sinensis roots. To sum up, $+\mathrm{Al} C$. sinensis seedlings had higher capacity to maintain the cellular $\mathrm{P}$ homeostasis via enhancing $\mathrm{P}$ acquisition and utilization, hence protecting plants against Al-toxicity. This agrees with our data that leaf, stem and root $\mathrm{P}$ level, and $\mathrm{P}$ uptake per plant were higher in $+\mathrm{Al} C$. sinensis than in $+\mathrm{Al}$ C. grandis seedlings (Figures 2C,D; Yang L.T. et al., 2011).

\section{Genes Related to S Transport and Metabolism}

S-mediated alleviation of Al-toxicity has been found in barley (Dawood et al., 2012) and wheat (Zhang et al., 2010). Here, we isolated one upregulated sulfate transporter (SULTR) 3,5 isoform 1 gene and two downregulated genes encoding SULTR 1,3 isoform 1 and SULTR 3.1-like, two upregulated sulfate/bicarbonate/oxalate exchanger and transporter sat-1 genes, and two upregulated sulfite exporter TauE/SafE family protein genes from $+\mathrm{Al} C$. grandis roots, but only two upregulated genes encoding SULTR 3,5 isoform 1 and SULTR 3.1-like and one downregulated sulfite exporter TauE/SafE family protein from $+\mathrm{Al}$ C. sinensis roots (Supplementary Table S7). Thus, $\mathrm{S}$ uptake and export might be increased and reduced in $+\mathrm{Al}$ C. sinensis seedlings, respectively, thus enhancing S level and the Al-tolerance of $C$. sinensis. This was also supported by our data that $S$ level was higher in C. sinensis roots and leaves than in C. grandis ones at each given Al level except for a similar root $\mathrm{S}$ level between the two species at the absence of $\mathrm{Al}$ (Figures 2E,F). $\mathrm{S}$ metabolism is the kernel pathway for the biosynthesis of molecules necessary for plant growth and development as well as tolerance to environmental stresses including $\mathrm{Al}$ (Yang et al., 2007; Anjum et al., 2015; Jiang et al., 2015). Here, we identified more downregulated (25) than upregulated (13) genes from $+\mathrm{Al}$ C. grandis roots, but less downregulated (9) than upregulated (14) genes related to $\mathrm{S}$ metabolism from $+\mathrm{Al} C$. sinensis roots (Supplementary Table S7). Based on these results, we concluded that genes involved in $\mathrm{S}$ transport and metabolism might contribute to the Al-tolerance of $C$. sinensis by elevating cell $\mathrm{S}$ level and increasing the biosynthesis of S-containing compounds responsible for Al detoxification.

\section{Genes Related to Antioxidation}

$\mathrm{Al}$ can elicit the production of ROS in plant cells (Yin et al., 2010; Li et al., 2016). Plants have developed diverse mechanisms for the detoxification of ROS. Besides the upregulation of some $\mathrm{S}$ metabolism-related genes, other genes related to antioxidation might be altered in $+\mathrm{Al}$ citrus roots. Here, we isolated 17 upregulated antioxidant enzyme genes (i.e., 11 PODs, five GLPs and one peroxiredoxin-2B-like) and three downregulated GLP 9-3 from $+\mathrm{Al} C$. sinensis roots. By contrast, we obtained 14 upregulated (i.e., five PODs and nine GLPs) and eight downregulated (i.e., four PODs and four GLP 9-3) genes from $+\mathrm{Al}$ C. grandis roots (Supplementary Table S8). Generally viewed, the expression of antioxidant enzyme genes was upregulated in $+\mathrm{Al}$ C. sinensis and C. grandis roots, especially in $+\mathrm{Al}$ C. sinensis roots. This agrees with our report that the levels of proteins related to ROS scavenging were increased in $+\mathrm{Al} C$. sinensis roots, but were less altered in + $\mathrm{Al}$ C. grandis roots (Jiang et al., 2015).

\section{Genes Related to Polysaccharide, Cell Wall and Cytoskeleton Metabolism}

Root cell wall is the key target for Al-toxicity and Al-tolerance (Horst et al., 2010). Here, we isolated 90 downregulated and 28 upregulated, and 25 downregulated and 39 upregulated genes related to polysaccharide and cell wall metabolism from $+\mathrm{Al}$ C. grandis and C. sinensis roots, respectively (Supplementary Table S9), implying that polysaccharide and cell wall metabolism was less impaired in $+\mathrm{Al} C$. sinensis than in $+\mathrm{Al} C$. grandis roots.

The Al-induced biosynthesis of root callose is an indicator of Al-injury and a reliable index for the evaluation of Al-tolerance (Horst et al., 2010). The upregulation of callose synthase 5-like in $+\mathrm{Al}$ C. grandis roots also support the above inference that C. grandis had less Al-tolerance than C. sinensis. In addition, we isolated six downregulated and one upregulared cellulose synthases from $+\mathrm{Al} C$. grandis roots, but only one downregulated and one upegulated cellulose synthases from $+\mathrm{Al} C$. sinensis roots. This agrees with the report that the Al-induced decrease in cellulose synthesis was less severe in Al-tolerant than in Alsensitive wheat cultivar. The reduction in cellulose synthesis has been suggested to be the cause responsible for the rapid inhibition of root elongation under Al-stress (Teraoka et al., 2002). The Alinduced downregulation of cellulose synthases also agrees with our result that callose synthase 5-like was upregulated in $+\mathrm{Al}$ C. grandis roots, because the Al-induced inhibition of cellulose synthesis has been suggested to be in favor of callose synthesis (Teraoka et al., 2002).

The interaction of $\mathrm{Al}$ with cell wall pectin may cause the stiffening of cell walls, thus inhibiting root cell elongation (Tabuchi and Matsumoto, 2001). Pectinesterases (PEs) catalyze the deesterification and deacetylation of pectin, and potentially regulate the degradation of cell wall via modifing the accessibility of pectin to the action of cell wall degrading enzymes (Chandran et al., 2008). Thus, PEs may improve cell wall loosening, thereby compensating for the Al-induced cell wall stiffening. Here, we obtained 16 downregulated genes involved in pectin deesterification (Cs1g16550, Cs1g16560, Cs5g33450, Cs3g06900, Cs4g06650, Cs4g06630, Cs4g06670, Cs4g06690 and orange1.1t02719), degradation (Cs8g11330, orange1.1t01738 and Cs7g21940), biosynthesis (Cs2g01430, Cs5g33680 and Cs3g24380) and depolymerization (Cs2g10560) and three upregulated genes (Cs2g16380, Cs7g16400 and orange1.1t00214) involved in pectin biosynthesis from $+\mathrm{Al} C$. grandis roots, but all the four DEGs (orange1.1t00214, orange1.1t01727, Cs3g06900 and Cs5g33420) related to pectin deesterification were 
upregulated in $+\mathrm{Al} C$. sinensis roots. Thus, the elevated mRNA levels of four genes might contribute to the higher Al-tolerance of $C$. sinensis.

The modifications of the cellulose-xyloglucan network (primary cell walls) are required for plant cell expansion (Yang J.L. et al., 2011). The modifications of primary cell walls are catalyzed by various enzymes, including xyloglucan endotransglucosylase/hydrolase (XTH) family protein. XTHs catalyze either endotransglycosylation of xyloglucan via xyloglucan endotransglucosylase (XET) activity and/or the hydrolysis of xyloglucan via xyloglucan endohydrolase (XEH) activity, thereby contributing to cell wall loosing (Chandran et al., 2008). Yang J.L. et al. (2011) showed that the Al-induced inhibition of XET activity was the key process causing the inhibition of root growth under Al-stress. Here, we identified nine downregulated XTH (XET) and three downregulated genes encoding xyloglucanspecific endoglucanase inhibitor protein from $+\mathrm{Al}$ C. grandis roots, but only one upregulated xyloglucanspecific endoglucanase inhibitor protein from $+\mathrm{Al} C$. sinensis roots, suggesting that the downregulation of XTH (XET) played a role in the Al-induced inhibition of $C$. grandis roots. This agrees with our data that Al-treatment only reduced C. grandis root DW (Figure 1A), and that callose synthase 5-like was upregulated only in $+\mathrm{Al} C$. grandis roots because the Al-induced inhibition of XET activity and the deposition of callose in Arabidopsis roots was paralleled (Yang J.L. et al., 2011).

Expansins, which serve as wall-loosening proteins, play a role in plant growth and response to abiotic stresses (Cosgrove, 2015). As shown in Supplementary Table S9, we isolated five downregulated expansins from $+\mathrm{Al} C$. grandis roots, but four upregulated and five downregulated expansins from $+\mathrm{Al}$ C. sinensis roots. Also, we isolated two downregulated extensins involved in root hair morphogenesis and elongation (Baumberger et al., 2001) from +Al C. grandis roots. Thus, the downregulation of expansins and extensins might play a role in the Al-induced inhibition of root growth and Al-sensitivity in C. grandis seedlings. However, recent work showed that an Alinducible expansin gene, OsEXPA10 was necessary for normal root growth, but its contribution to the high Al-tolerance of rice was small (Che et al., 2016).

Ascorbate oxidase (AO) has been suggested to increase cell elongation via producing monodehydroascorbate and dehydroascorbate (Pignocchi et al., 2003). Our results showed that L-ascorbate oxidase homolog was inhibited and induced in $+\mathrm{Al}$ C. grandis and C. sinensis roots, respectively, which agrees with our data that root growth inhibition only occurred in $+\mathrm{Al}$ C. grandis (Figure 1A).

In addition to scavenging $\mathrm{H}_{2} \mathrm{O}_{2}$, PODs also produce $\mathrm{H}_{2} \mathrm{O}_{2}$ at the expense of $\mathrm{NAD}(\mathrm{P}) \mathrm{H}$. The total activities of PODs increase when exposed to $\mathrm{Al}$ (Cakmak and Horst, 1991). The exact roles of the Al-induced increase in PODs-mediated $\mathrm{H}_{2} \mathrm{O}_{2}$ production in roots are unclear (Maron et al., 2008). PODs play a role in the oxidative cross-linking of cell wall compoments, thus increasing cell wall stiffening and decreasing cell wall extensibility, which has been associated with the Al-induced inhibiton of root growth ( $\mathrm{Ma}$ et al., 2012). POD-mediated oxidative cross-linking, however, has been demonstrated to be a potential mechanism of Al-tolerance
(Ma et al., 2004; Maron et al., 2008). Plants may use oxalate oxidase (OXO)- and/or POD-dependent production of $\mathrm{H}_{2} \mathrm{O}_{2}$ to restructure the cell walls and restrict $\mathrm{Al}$ entry via decreasing cell wall porosity (Delisle et al., 2001; Maron et al., 2008). Tamás et al. (2005) showed that the Al-induced cell death of barley-root border cells due to the increase in POD- and OXOmediated production of $\mathrm{H}_{2} \mathrm{O}_{2}$ played a role in protecting root tips against Al-toxicity via chelating $\mathrm{Al}$ in the dead cells. Here, we identified five upregulated and four downregulated PODs from $+\mathrm{Al}$ C. grandis roots, but 11 upregulated $P O D$ from $+\mathrm{Al}$ C. sinensis roots, indicating that PODs might be involved in the Al-tolerance of $C$. sinensis. Similarly, the expression levels of all genes encoding the germin-like proteins (GLPs), the $\mathrm{H}_{2} \mathrm{O}_{2}$ generating enzymes with $\mathrm{OXO}$ and superoxide dismutase (SOD) activities, were increased in $+\mathrm{Al} C$. sinensis and C. grandis roots except for three and four downregulated GLP 9-3 in $+\mathrm{Al}$ C. sinensis and C. grandis roots, respectively. Maron et al. (2008) observed that the expression levels of OXOs were constitutively higher and were increased by $\mathrm{Al}$ only in Al-tolerant maize roots. Thus, GLPs might play a role in the higher Al-tolerance of C. sinensis.

Cytoskeleton has been demonstrated to be a target of Altoxicity in plants. In the early response to $\mathrm{Al}$, a close relationship between the root growth inhibition and the impairments of the integrity of cytoskeletal elements (microtubules and actin microfilaments) was observed, specifically in the distal part of the transition zone in the Al-sensitive maize (Sivaguru et al., 1999). Here, we isolated four downregulated (Cs5g03620, Cs8g03680, Cs4g12990, and Cs8g09980) and two upregulated (Cs8g09980 and Cs1g15130) genes related to cytoskeleton metabolism from $+\mathrm{Al}$ C. grandis roots, indicating that cytoskeleton might be impaired in roots, thereby inhibiting root growth. By contrast, we isolated 11 upregulated and one dowregulated cytoskeleton metabolism-related genes from $+\mathrm{Al} C$. sinensis roots.

These results strongly suggested that Al-treatment impaired polysaccharide, cell wall and cytoskeleton metabolism in C. grandis roots, thus inhibiting root growth and lowering Altolerance.

\section{Genes Related to Carbohydrate and Energy Metabolsim}

Energy shortage is a common phenomenon for stressed plants. A close relation exists between stress tolerance and energy availability (Baena-González and Sheen, 2008). As shown in Supplementary Table S10, we isolated 6 downregulated (Cs2g03260, Cs8g06950, orange1.1t05835, Cs3g22480, Cs2g13410 and Cs5g30520) and two upregulated (Cs4g08150 and Cs4g01490) genes related to aerobic glycolysis from $+\mathrm{Al}$ C. grandis roots, but only six upregulated genes (Cs5g30520, Cs4g01490, Cs1g15970, Cs4g13070, Cs3g18540 and Cs6g08840) from $+\mathrm{Al} C$. sinensis roots. Thus, glycolysis might be repressed in $+\mathrm{Al}$ C. grandis roots, but induced in $+\mathrm{Al} C$. sinensis roots. Wang et al. (2014) found that the majority of the Al-responsive proteins related to glycolysis were upregulated in Al-tolerant rice cultivar. Thus, the higher glycolysis in $+\mathrm{Al} C$. sinensis roots might contribute to the Al-tolerance of $C$. sinensis by keeping 
basic root respiration and meeting the increased requirement for engery (Wang et al., 2014). Interestingly, the mRNA level of phosphoenolpyruvate carboxykinase (PEPCK) [ATP]-like (a key regulatory enzyme in gluconeogenesis) gene was repressed in $+\mathrm{Al}$ C. sinensis roots. This agrees with the report that PEPCK was severely reduced in Al-stressed Pseudomonas fluorescens cells (Lemire et al., 2008). PEPCK plays a role in the catabolism of malate/citrate during fruit ripening (Baldicchi et al., 2015). Malate level was enhanced in the ripening fruits of PEPCK-RNAi tomato lines relative to the wild type (Huang et al., 2015). Thus, the down-regulation of PEPCK [ATP]-like might promote malate/citrate accumulation in $+\mathrm{Al} C$. sinensis roots. By contrast, the expression of NADP-malic enzyme 4 (NADP-ME4) was upregulated in $+\mathrm{Al}$ C. sinensis roots. Overexpression of an $\mathrm{Al}-$ induced ME gene (SgME1) from Stylosanthes increased malate synthesis and prevented yeast, Arabidopsis and bean hairy roots from Al-toxicity through increased root malate synthesis and/or accompanied root malate exudation (Sun et al., 2014). Thus, root levels or secretion of malate/citrate might be enhanced due to decreased expression of PEPCK and increased expression of NADP-ME4, hencing conferring Al-tolerance of C. sinensis. This agrees with our report that $+\mathrm{Al}$ C. sinensis seedlings had higher or similar root levels of malate, citrate and malate + citrate and increased root secretion of malate and citrate than +Al C. grandis ones (Figure 3; Yang L.T. et al., 2011), suggesting that $C$. sinensis roots might have a more efficient chelation system, thus protecting roots against Al-toxicity. In addition, the Al-induced alterations of other genes related to energy and carbohydrate metabolism differed between the two citrus species. For example, we isolated two upregulated alcohol dehydrogenase $(A D H)$ and one downregulated $A D H$ class-3 $(A D H-3)$ related to anaerobic glycolysis from $+\mathrm{Al} C$. sinensis roots. This agrees with the reports that $\mathrm{ADH}$ activity was greatly increased in $\mathrm{Al}$-treated wheat roots (Copeland and De Lima, 1992). Study showed that STOP1 regulated multiple gene expression, thus protecting plants against $\mathrm{H}^{+}$and $\mathrm{Al}$ toxicities. Genes involved in $\mathrm{pH}$-regulating metabolism such as $M E$, pyruvate decarbohylase (PDC) and $A D H$ [or lactate dehydrogenase $(L D H)$ ] were downregulated in stop1 mutant (Sawaki et al., 2009). Thus, the Al-induced upregulation of ADH, NADP-ME4 and thiamine pyrophosphate dependent pyruvate decarboxylase family protein might contribute to the Al-tolerance of C. sinensis via keeping the $\mathrm{pH}$ homeostasis of cells. In short, we obtained more upregulated (18) than downregulated (10) genes related to energy and carbohydrate metabolism from $+\mathrm{Al}$ C. sinensis roots, but more downregulated (19) than upregulated (9) genes from $+\mathrm{Al}$ C. grandis roots. The energy and carbohydrate metabolism displayed higher adaptive responses to $\mathrm{Al}$ in C. sinensis than in C. grandis roots (Jiang et al., 2015).

\section{Genes Related to Protein and Amino Acid Metabolism}

We isolated 88 and $56 \mathrm{Al}$-responsive genes related to protein metabolism from C. grandis and C. sinenis roots, respectively (Supplementary Table S11), which might be related to the higher Al-tolerance of C. sinensis. This disagrees with our reports that more Al-responsive proteins related to protein metabolism were identified in C. sineneis than in C. grandis roots (Jiang et al., 2015). Most of these genes were involved in protein degradation, followed by protein ubiquitination-related genes, heat shock protein (HSP)/chaperone genes and protein biosynthesis-related genes. However, our previous study showed that over $50 \%$ of Alresponsive genes related to protein metabolism were involved in protein biosynthesis in C. sinensis and C. grandis roots. The differences between the Al-responsive proteins obtained by iTRAQ (Jiang et al., 2015) and the DEGs revealed by RNA-Seq might be due to PTMs.

Protein degradation can not only supply respiratory substrates for stressed plants, but also trigger adaptive responses to stressed conditions by nutrient recycling (Araújo et al., 2011). Proteases (also called peptidases and proteinases) play crucial roles in the selective degradation of specific proteins and the strict control of protein quality in plants when exposed to stressed conditions. The ubiquitin proteosome pathway may degradate futile and inactive proteins in a more specific manner relative to proteases (Kumari et al., 2008). Here, we identified 45 downregulated and 26 upregulated, and 28 downregulated and 18 upregulated genes related to protein degradation in $+\mathrm{Al} \mathrm{C}$. grandis and C. sinensis roots, respectively. The more downregulation of protein degradation-related genes might be responsible for the less Al-tolerance of C. grandis.

Amino acids play important roles in plant responses to different stresses. Here, we isolated eight upregulated and four downregulated, and 21 downregulated and four upregulated amino acid metabolism-related genes from $+\mathrm{Al}$ C. sinensis and C. grandis roots, respectively (Supplementary Table S11), demonstrating that amino acid metabolism might be involved in the higher Al-tolerance of C. sinensis.

\section{Genes Related to Lipid Metabolism}

The Al-induced alterations of the total and the relative abundance of lipids (particularly phospholipids) as well as the fatty acid composition and the degree of fatty acid unsaturation differed between Al-tolerant and Al-sensitive cultivars (Peixoto et al., 1999; Huynh et al., 2012). Huynh et al. (2012) reported that phospholipids and MGDG in roots decreased in Al-sensitive rice cultivars when exposed to $\mathrm{Al}$, while the amount of lipid classes kept stable in Al-tolerant ones. Here, we isolated 39 downregulated and 19 upregulated, and 12 downregulated and 28 upregulated genes related to metabolisms of lipids and fatty acids from $+\mathrm{Al} \mathrm{C.} \mathrm{grandis} \mathrm{and} \mathrm{C.} \mathrm{sinensis} \mathrm{roots,} \mathrm{respectively}$ (Supplementary Table S12), demonstrating that lipid metabolism was less affected by $\mathrm{Al}$ in $C$. sinensis roots, and that lipid metabolism might be upregulated and downregulated in $+\mathrm{Al}$ C. sinensis and C. grandis roots, respectively. This might be involved in the Al-tolerance of C. sinensis.

All the four DEGs related to jasmonic acid (JA) biosynthesis were upregulated in $+\mathrm{Al} C$. sinensis roots, but only two upregulated lipoxygenase (LOX) and two downregulated [ LOX3 and allene oxide synthase (AOS)] genes were isolated from $+\mathrm{Al}$ C. grandis roots (Supplementary Table S12). This agrees with the report that both JA biosynthesis and level might be elevated in +Al C. sinensis roots (Jiang et al., 2015). Peixoto et al. (2001) 
reported that both LOX activity and Al-induced increase in LOX activity were greater in Al-tolerant than in Al-sensitive sorghum cultivar. Therefore, JA metabolism might be upregulated in $+\mathrm{Al}$ C. sinensis roots, thereby contributing to the higher Al-tolerance of $C$. sinensis.

GDSL esterases/lipases play key role in plant abiotic stresses. Overexpression of LTL1 encoding a GDSL-motif lipase conferred the tolerance of salt and other environmental stresses in transgenic yeast and Arabidopsis plants (Jiang et al., 2012; Naranjo et al., 2006). Here, we identified four upregulated and two downregulated, and four downregulated GDSL lipases in $+\mathrm{Al}$ C. sinensis and C. grandis roots, respectively, demonstrating the possible involvement of GDSL lipases in citrus Al-tolerance.

We isolated 11 downregulated genes (Cs4g04540, Cs6g22090, orange1.1t02015, Cs2g06240, Cs3g15530, Cs3g20840, Cs4g17260, Cs5g01990, Cs6g08600, orange1.1t02024 and Cs4g06470) from + Al C. grandis roots, and four upregulated (Cs3g20840, orange1.1t00556, Cs8g20310 and Cs4g06430) and one downregulated (Cs4g06470) genes involved in fatty acid biosynthesis from $+\mathrm{Al}$ C. sinensis roots (Supplementary Table S12). Thus, fatty acid biosynthesis might be downregulated and upregulated in $+\mathrm{Al}$ C. grandis and C. sinensis roots, respectively. This agrees with the report that the levels of linolenic and palmitic acids, which comprised together about $65-71 \%$ of total fatty acids in the root plasma membrane fraction, increased in the Al-tolerant sorghum cultivar roots but decreased in the Al-sensitive ones (Peixoto et al., 2001).

Zhang et al. (2016) demonstrated that overexpression of OsMGD conferred Al-tolerance in transgenic tobacco plants via the regulation of galactolipid biosynthesis. However, the different Al-tolerance between the two citrus species could not be explained in this way because the Al-induced upregulation of MGD2 was greater in C. grandis than in C. sinensis roots.

\section{Genes Related to Signal Transduction}

As shown in Supplementary Table S13, 104 downregulated and 70 upregulated, and 55 downregulated and 43 upregulated genes related to signal transduction were isolated from $+\mathrm{Al}$ C. grandis and C. sinensis roots, respectively. Over twothirds of these DEGs belonged to protein kinases, followed by DEGs in $\mathrm{Ca} /$ calmodulim- and hormone-medicated signal transduction, and protein dephosphorylation. For the optimal regulation, a proper balance must be struck between protein kinases and phosphatase in any given plant cell. Jiang et al. (2015) demonstrated that Al-tolerant C. sinensis roots could keep a better balance between phosphorylation and dephosphorylation than C. grandis roots when exposed to Al. Here, we isolated 76 downregulated and 54 upregulated genes related to protein phosphorylation, and five downregulated and three upregulated genes related to dephosphorylation from $+\mathrm{Al}$ C. grandis roots, but only 36 downregulated and 29 upregulated genes related to protein phosphorylation, and two upregulated and two downregulated genes related to dephosphorylation from $+\mathrm{Al} C$. sinensis roots. Root protein phosphorylation/dephosphorylation was less affected in $+\mathrm{Al}$ C. sineneis roots. This might be associated with the Altolerance of $C$. sinensis. However, the exact roles of these kinases and phosphatases require further investigation. For example, Sivaguru et al. (2003) showed that overexpression of an Alinducible wall-associated receptor kinase 1 (WAK1) conferred Altolerance in transgenic Arabidopsis, suggesting that WAK1 was one of the key candidates for Al-tolerance. Here, we identified seven upregulated and three downregulated WAKs in $+\mathrm{Al}$ C. grandis roots, but only four downregulated WAKs in $+\mathrm{Al}$ C. sinensis roots. Therefore, the difference in the Al-tolerance between the two citrus species can not be explained in this way.

Disruption of cytoplasmic Ca homeostasis has been proposed to be a primary trigger of Al-toxicity (Rengel and Zhang, 2003). Okekeogbu et al. (2014) found that the abundances of several Ca-binding proteins were elevated in the Al-treated tomato radicles, suggesting that $\mathrm{Ca}$-binding proteins might play a role in tomato Al-tolerance. Here, we identified seven upregulated and two downregulated genes involved in $\mathrm{Ca} /$ calmodulim-medicated signal transduction in $+\mathrm{Al} C$. sinensis roots, demonstrating that $\mathrm{Al}$ might trigger $\mathrm{Ca} /$ calmodulim-medicated signal pathways in these roots, thereby contributing to the Al-tolerance of C. sinensis. By contrast, we obtained 12 downregulated and four upregulated genes related to $\mathrm{Ca} /$ calmodulim-medicated signal transduction from $+\mathrm{Al} C$. grandis roots, indicating that $\mathrm{Al}$ might impair $\mathrm{Ca} /$ calmodulim-medicated signal pathways in these roots, thus lowering the Al-tolerance of C. grandis.

Plant hormones, which play crucial roles in signal transduction, are involved in Al-tolerance. As shown in Supplementary Table S13, all the 10 DEGs related to hormonemediated signal transduction were upregulated in $+\mathrm{Al}$ C. sinensis roots, indicating that $\mathrm{Al}$ might activate hormone-mediated signal pathways, thus enhancing C. sinensis Al-tolerance. By contrast, we isolated six downregulated and three upregulated genes from $+\mathrm{Al}$ C. grandis roots, implying that hormone-mediated signal pathways might be disturbed in these roots, thus affecting the Al-tolerance of C. grandis.

\section{Transcription Factors}

As shown in Supplementary Table S14, 51 downregulated and 44 upregulated, and 37 downregulated and 33 upregulated TFs were identified in $+\mathrm{Al}$ C. grandis and C. sinensis roots, respectively. Most of them belonged to myeloblastosis (MYB), Apetala2 (AP2)/ethylene-responsive transcription factor (ERF), zinc finger [C-x8-C-x5-C-x3-H (CCCH) and Cys2/His2 (C2H2)], WRKY, homeobox, helix-loop-helix (bHLH) and NAC families. Similar results have been obtained on Al-treated soybean (You et al., 2011) and Arabidopsis (Kumari et al., 2008) roots.

STOP1, a $\mathrm{C} 2 \mathrm{H} 2$-zinc-finger protein, played a key role in $\mathrm{H}^{+}$- and Al-tolerance (Sawaki et al., 2009). However, this could not explain the difference in the Al-tolerance between the two citrus species, because the Al-induced upregulation of two protein SENSITIVE TO PROTON RHIZOTOXICITY 1-like genes was greater in C. grandis than in C. sinensis roots. Also, ART1, a C2H2-type zinc-finger TF, has been demonstrated to regulate the expression of multiple Al-tolerance genes required for rice Al-tolerance (Yamaji et al., 2009). Here, we isolated one downregulated $\mathrm{C} 2 \mathrm{H} 2$-type zinc finger protein and one upregulated $\mathrm{C} 2 \mathrm{H} 2$-like zinc finger protein from Al-treated C. grandis and C. sinensis roots, respectively, implying that $\mathrm{C} 2 \mathrm{H} 2$ 
One $\uparrow$ CalS5L, six $\downarrow$ and one $\uparrow$ CelS in +Al CGR; One $\uparrow$ CalS5L, one $\downarrow$ and one $\uparrow$ CelS in +Al CSR; Sixteen $\downarrow$ and three $\uparrow$ genes in pectin metabolism in +Al CGR, four $\uparrow$ genes in +Al CSR;

Nine $\downarrow$ XTH(XET) and three $\downarrow$ XEIP in +AI CGR, one $\uparrow$ XEIP in +Al CSR;

Five $\downarrow$ EXPs, two $\downarrow$ EXTs and one $\downarrow$ AOH in +Al CGR, four $\uparrow$ and five $\downarrow$ EXPs and one $\uparrow \mathrm{AOH}$ in $+\mathrm{AI}$ CSR; Four $\downarrow$ and two $\uparrow$ genes in cytoskeleton in +Al CGR, $11 \uparrow$ and one $\downarrow$ genes in + Al CSR.

To conclude, $90 \downarrow$ and $25 \uparrow$ genes related to PCWCM in $+\mathrm{Al} \mathrm{CGR}$, and $25 \downarrow$ and $38 \uparrow$ genes in +Al CSR.

Cell wall and cytoskeleton metabolism, energy and carbohydrate metabolism and signal transduction displayed higher adaptive responses to $\mathrm{Al}$ in CSR than in CGR.

\begin{tabular}{|l|}
\multicolumn{1}{c|}{} \\
\hline Six $\downarrow$ and two $\uparrow$ genes \\
related to glycolysis \\
in +Al CGR, six $\uparrow$ \\
genes in +Al CSR; \\
One $\downarrow$ PEPCK.ATP]- \\
like and one $\uparrow$ NADP- \\
ME4 in +Al CSR; \\
To conclude, 18 $\uparrow$ and 10 $\downarrow$ \\
genes in carbohydrate \\
and energy metabolism \\
in +Al CSR, nine $\uparrow$ and $19 \downarrow$ \\
genes in +Al CGR. \\
\hline
\end{tabular}

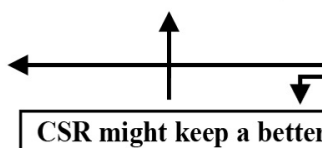

balance between phosphorylation and dephosphorylation than CGR when exposed to $\mathrm{Al}$; Both Ca/Calmodulimand hormone-mediated signal pathways might be activated in +Al CSR, but impaired in +Al CGR.

Five $\uparrow$ GLPs, $11 \uparrow$ PODs, one $\uparrow \mathrm{P} 2 \mathrm{~L}$ and three $\downarrow$ GLP 9-3 in +Al CSR;

Antioxidant capacity might be enhanced in +AI CSR and CGR, especially in +AI CSR. $\leftarrow$ Nine $\uparrow$ GLPs, five $\uparrow$ PODs, four $\downarrow$ PODs and four $\downarrow$ GLP 9-3 in +Al CGR.
4

Stem, leaf and root $P$ level, and $P$ uptake per seedling were higher in $+\mathrm{Al}$ CS than in +Al CG seedlings; Al-induced upregulation of low-Presponsive genes was greater in CSR than CGR.

\section{CSR secreted more malate and citrate than CGR when exposed to $\mathrm{Al}$; Less Al was accumulated in $+\mathrm{Al}$ CS leaves than in +Al CG leaves; $+\mathrm{Al}$ CSR had higher or similar levels of malate, citrate and malate + citrate than +Al CGR. I} via enhanced $\mathrm{Al}$-induced secretion of $O A$ anions, and a more efficient chelation system in roots.

+ Al CS seedlings had a higher external Al detoxification capacity

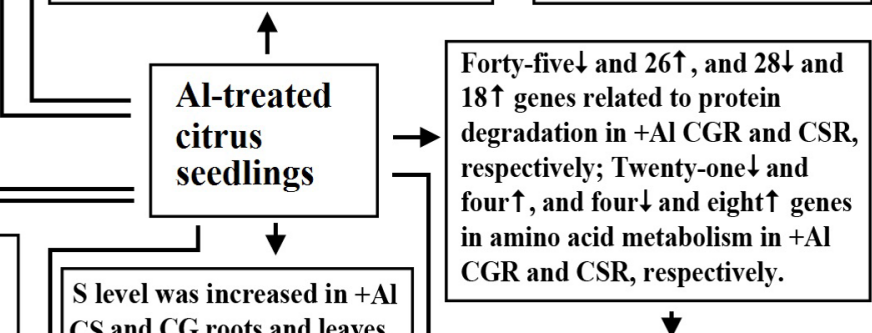
$C S$ and $C G$ roots and leaves, especially in +AlCS roots and leaves; $S$ uptake-related genes were induced, and $S$ export-related genes were inhibited in +Al CSR; More upregulated than downregulated $S$ metabolismrelated genes was obtained in $+\mathrm{Al}$ CSR, but the reverse was the case in +Al CGR.

\section{$\downarrow$}

Genes in S transport and metabolism might play a role in CS Al-tolerance via enhancing cell $S$ level and the biosynthesis of S-containing compounds responsible for $\mathrm{Al}$ detoxification.

FIGURE 6 | A potential responses of citrus seedlings to Al. AOH, ascorbate oxidase homolog; CG, C. grandis; CGR, C. grandis roots; CS, C. sinensis; CSR, C. sinensis roots; EXPs, expansins; EXTs, extensins; PCWCM, polysaccharide, cell wall and cytoskeleton metabolism; XEIP, xyloglucan-specific endoglucanase inhibitor protein.

zinc finger TFs might be involved in the higher Al-tolerance of C. sinensis.

Arenhart et al. (2014) found that the expression level of $A B A$ stress ripening 5 (ASR5) was not altered in the Al-sensitive rice cultivar roots when exposed to $\mathrm{Al}$, but was upregulated in the Al-tolerant ones. Further analysis demonstrated that ASR5 played a key role in Al-tolerance by mediating Al-responsive gene expression. Here, we identified three upregulated genes encoding ASR-related protein from $+\mathrm{Al}$ C. grandis roots, but only one downregulated ASR-related genes from $+\mathrm{Al} C$. sinensis roots. Roselló et al. (2015) indicated that that Al increased ASR1 expression level up to six fold in the roots of Al-sensitive rice cultivar, but not in Al-tolerant cultivar. Further studies are needed to elucidate the roles of ASR genes in plant Al-tolerance.
Plant WRKY TFs have been shown to play important roles in the responses to abiotic stresses including Al-toxicity (Chen L. et al., 2012). Mattiello et al. (2010) found that a member of the WRKY transcriptional family was upregulated only in Al-tolerant maize line roots when grown in acidic soil with elevated $\mathrm{Al}$ level. Here, we isolated two upregulated and four downregulated WRKYs from + Al C. grandis roots, and four upregulated and two downregulated WRKYs from $+\mathrm{Al} C$. sinensis roots. The Al-induced upregulation of WRKYs might play a role in the Al-tolerance of C. sinensis. However, Ding et al. (2013) showed that WRKY46 was repressed by $\mathrm{Al}$ and negatively regulated the expression of ALMT1. Mutation of WRKY46 led to increased Al-tolerance by enhancing Al-induced secretion of malate and decreasing $\mathrm{Al}$ accumulation in root apices. 
MYB TFs, especially the large family of plant-specific R2R3MYB genes play a role in plant responses to environmental stresses. Dai et al. (2012) observed that the expression of OsMYB2P-1, an R2R3 MYB TF, was induced by P-deficiency, mainly in rice roots and stems, and that transgenic Arabidopsis and rice plants overexpressing OsMYB2P-1 had enhanced tolerance to $\mathrm{P}$-deficiency, while OsMYB2P-1 RNAi transgenic rice plants were more sensitive to $\mathrm{P}$-deficiency relative to wild-type plants. Here, we identified seven upregulated and three downregulated MYBs in $+\mathrm{Al} C$. sinensis roots, and nine upregulated and 11 downregulated $M Y B s$ in $+\mathrm{Al}$ C. grandis roots. It is worth mentioning that the expression of $R 2 R 3-M Y B T F$ and R2R3 TF MYB108-like protein 1 was upregulated in $+\mathrm{Al}$ C. sinensis roots, while the expression of R2R3 TF MYB108like protein 1 and $R 2 R 3-M Y B$ TF was downregulated in $+\mathrm{Al}$ C. grandis roots. Thus, the Al-induced upregulation of MYB TFs might contribute to the Al-tolerance of $C$. sinensis via enhancing the tolerance to P-starvation.

To conclude, the great difference in the Al-induced alterations of TF expression profiles in the two citrus species implies that TFs might play a role in the Al-tolerance of $C$. sinensis.

\section{CONCLUSION}

Using RNA-seq, we isolated 1905 and 2670 Al-responsive genes from C. sinensis and C. grandis roots, respectively. Among these DEGs, only 649 DEGs were shared by the both. Through the integration of the present findings and the available data in the previous reports, a model for the adaptive responses of citrus seedlings to $\mathrm{Al}$ was proposed (Figure 6). There were common and unique mechanisms for Al-tolerance in citrus plants. The following several aspects might account for the higher Altolerance of $C$. sinensis: (a) $+\mathrm{Al} C$. sinensis seedlings displayed a more efficient exclusion mechanism, a higher antioxidant capacity and a more efficient chelation system in roots; (b) $+\mathrm{Al} C$. sinensis seedlings had higher capacity to maintain the cellular $\mathrm{P}$ homeostasis via improving $\mathrm{P}$ acquisition and utilization; (c) Genes in $\mathrm{S}$ transport and metabolism might contribute the Al-tolerance of C. sinensis via enhancing cell S level

\section{REFERENCES}

Ames, B. N. (1966). Assay of inorganic phosphate, total phosphate and phosphatase. Methods Enzymol. 8, 115-118. doi: 10.1016/0076-6879(66) 08014-5

Anjum, N. A., Gill, R., Kaushik, M., Hasanuzzaman, M., Pereira, E., Ahmad, I., et al. (2015). ATP-sulfurylase, sulfur-compounds, and plant stress tolerance. Front. Plant Sci. 6:210. doi: 10.3389/fpls.2015.00210

Araújo, W. L., Tohge, T., Ishizaki, K., Leaver, C. J., and Fernie, A. R. (2011). Protein degradation-an alternative respiratory substrate for stressed plants. Trends Plant Sci. 16, 489-498. doi: 10.1016/j.tplants.2011.05.008

Arenhart, R. A., Bai, Y., de Oliveira, L. F., Neto, L. B., Schunemann, M., Maraschin, F., et al. (2014). New insights into aluminum tolerance in rice: the ASR5 protein binds the STAR1 promoter and other aluminum-responsive genes. Mol. Plant 7, 709-721. doi: 10.1093/mp/sst160

Baena-González, E., and Sheen, J. (2008). Convergent energy and stresssignaling. Trends Plant Sci. 13, 474-482. doi: 10.1016/j.tplants.2008.06.006 and the biosynthesis of S-containing compounds responsible for $\mathrm{Al}$ detoxification; (d) Al impaired polysaccharide, cell wall and cytoskeleton metabolism in C. grandis roots, thus lowering Al-tolerance; (e) Energy and carbohydrate metabolism, and signal transduction displayed higher adaptive responses to $\mathrm{Al}$ in $C$. sinensis than in C. grandis roots; (f) Genes involved in fatty acid, protein and amino acid metabolisms might be involved in the Al-tolerance of $C$. sinensis. To conclude, we first comparatively investigated the transcriptomic responses of citrus roots to $\mathrm{Al}$ using two citrus species with different $\mathrm{Al}$-tolerance.

\section{DATA ACCESS}

RNAseq are submitted to Gene Expression Omnibus (GEO) under accession no GSE85958 (http://www.ncbi.nlm.nih.gov/geo/ query/acc.cgi?acc = GSE85958).

\section{AUTHOR CONTRIBUTIONS}

PG carried out most of the experiments and drafted the manuscript. Y-PQ participated in the design of the study. L-TY participated in the design and coordination of the study. N-WL participated in the analysis of data. XY participated in the analysis of $\mathrm{Al}$ and $\mathrm{P}$. YY participated in the cultivation of seedlings. L-SC designed and directed the study and revised the manuscript. All authors have read and approved the final manuscript.

\section{FUNDING}

Our work was funded by the earmarked fund for China Agriculture Research System (No. CARS-27).

\section{SUPPLEMENTARY MATERIAL}

The Supplementary Material for this article can be found online at: http://journal.frontiersin.org/article/10.3389/fpls.2017.00330/ full\#supplementary-material

Baldicchi, A., Farinelli, D., Micheli, M., Di Vaio, C., Moscatello, S., Battistelli, A., et al. (2015). Analysis of seed growth, fruit growth and composition and phospoenolpyruvatecarboxykinase (PEPCK) occurrence in apricot (Prunus armeniaca L.). Sci. Hortic. 186, 38-46. doi: 10.1016/j.scienta.2015.01.025

Bariola, P. A., Howard, C. J., Taylor, C. B., Verburg, M. T., Jaglan, V. D., and Green, P. J. (1994). The Arabidopsis ribonuclease gene RNS1 is tightly controlled in response to phosphate limitation. Plant J. 6, 673-685. doi: 10.1046/j.1365-313X. 1994.6050673.x

Baumberger, N., Ringli, C., and Keller, B. (2001). The chimeric leucine-rich repeat/extensin cell wall protein LRX1 is required for root hair morphogenesis in Arabidopsis thaliana. Genes Dev. 15, 1128-1139. doi: 10.1101/gad. 200201

Bradford, M. M. (1976). A rapid and sensitive method for quantitation of microgram quantities of protein utilizing the principle of protein-dye binding. Anal. Biochem. 72, 248-254. doi: 10.1016/0003-2697(76)90527-3

Brunner, I., and Sperisen, C. (2013). Aluminum exclusion and aluminum tolerance in woody plants. Front. Plant Sci. 4:172. doi: 10.3389/fpls.2013.00172 
Cakmak, I., and Horst, W. J. (1991). Effect of aluminium on lipid peroxidation, superoxide dismutase, catalase, and peroxidase activities in root tips of soybean (Glycine max). Physiol. Plant. 83, 463-468. doi: 10.1111/j.1399-3054.1991. tb00121.x

Caparrós-Martín, J. A., McCarthy-Suárez, I., and Culiáñez-Macià, F. A. (2013). HAD hydrolase function unveiled by substrate screening: enzymatic characterization of Arabidopsis thaliana subclass I phosphosugar phosphatase AtSgpp. Planta 237, 943-954. doi: 10.1007/s00425-012-1809-5

Chandran, D., Sharopova, N., Ivashuta, S., Gantt, J. S., Vandenbosch, K. A., and Samac, D. A. (2008). Transcriptome profiling identified novel genes associated with aluminum toxicity, resistance and tolerance in Medicago runcatula. Planta 228, 151-166. doi: 10.1007/s00425-008-0726-0

Che, J., Yamaji, N., Shen, R. F., and Ma, J. F. (2016). An Al-inducible expansin gene, OsEXPA10 is involved in root cell elongation of rice. Plant J. 88, 132-142. doi: $10.1111 /$ tpj.13237

Chen, H., Lu, C., Jiang, H., and Peng, J. (2015). Global transcriptome analysis reveals distinct aluminum-tolerance pathways in the $\mathrm{Al}$-accumulating species Hydrangea macrophylla and marker identification. PLoS ONE 10:e0144927. doi: 10.1371 /journal.pone. 0144927

Chen, L., Song, Y., Li, S., Zhang, L., Zou, C., and Yu, D. (2012). The role of WRKY transcription factors in plant abiotic stresses. Biochim. Biophys. Acta 1819, 120-128. doi: 10.1016/j.bbagrm.2011.09.002

Chen, Z. C., Yamaji, N., Motoyama, R., Nagamura, Y., and Ma, J. F. (2012). Up-regulation of a magnesium transporter gene is required for conferring aluminum tolerance in rice. Plant Physiol. 159, 1624-1633. doi: 10.1104/pp.112. 199778

Cheng, Y., Zhou, W., El Sheery, N. I., Peters, C., Li, M., Wang, X., et al. (2011). Characterization of the Arabidopsis glycerophosphodiester phosphodiesterase (GDPD) family reveals a role of the plastid-localized AtGDPD1 in maintaining cellular phosphate homeostasis under phosphate starvation. Plant J. 66, 781-795. doi: 10.1111/j.1365-313X.2011.04538.x

Copeland, L., and De Lima, M. L. (1992). The effect of aluminum on enzyme activities in wheat roots. J. Plant Physiol. 140, 641-645. doi: 10.1016/S01761617(11)81017-5

Cosgrove, D. J. (2015). Plant expansins: diversity and interactions with plant cell walls. Curr. Opin. Plant Biol. 25, 162-172. doi: 10.1016/j.pbi.2015.05.014

Dai, X., Wang, Y., Yang, A., and Zhang, W. H. (2012). OsMYB2P-1, an R2R3 MYB transcription factor, is involved in the regulation of phosphate-starvation responses and root architecture in rice. Plant Physiol. 159, 169-183. doi: 10. 1104/pp.112.194217

Dawood, M., Cao, F., Jahangir, M. M., Zhang, G., and Wu, F. (2012). Alleviation of aluminum toxicity by hydrogen sulfide is related to elevated ATPase, and suppressed aluminum uptake and oxidative stress in barley. J. Hazard. Mater. 20, 121-128. doi: 10.1016/j.jhazmat.2011.12.076

Delhaize, E., Ryan, P. R., Hebb, D. M., Yamamoto, Y., Sasaki, T., and Matsumoto, H. (2004). Engineering high-level aluminum tolerance in barley with the ALMT1 gene. Proc. Natl. Acad. Sci. U.S.A. 101, 15249-15254. doi: 10.1073/pnas. 0406258101

Delisle, G., Champoux, M., and Houde, M. (2001). Characterization of oxalate oxidase and cell death in Al-sensitive and tolerant wheat roots. Plant Cell Physiol. 42, 324-333. doi: 10.1093/pcp/pce041

Deng, W., Luo, K., Li, D., Zheng, X., Wei, X., Smith, W., et al. (2006). Overexpression of an Arabidopsis magnesium transport gene, AtMGT $T_{1}$, in Nicotiana benthamiana confers Al tolerance. J. Exp. Bot. 57, 4235-4243.

Ding, Z. J., Yan, J. Y., Xu, X. Y., Li, G. X., and Zheng, S. J. (2013). WRKY46 functions as a transcriptional repressor of $A L M T 1$, regulating aluminuminduced malate secretion in Arabidopsis. Plant J. 76, 825-835. doi: 10.1111/tpj. 12337

Duff, S. M. G., Lefebvre, D. D., and Plaxton, W. C. (1989). Purification and characterization of a phosphoenolpyruvate phosphatase from Brassica nigra suspension cells. Plant Physiol. 90, 734-741. doi: 10.1104/pp.90.2.734

Fan, W., Lou, H. Q., Gong, Y. L., Liu, M. Y., Wang, Z. Q., Yang, J. L., et al. (2014). Identification of early Al-responsive genesin rice bean (Vigna umbellata) roots provides new clues to molecular mechanisms of Al toxicity and tolerance. Plant Cell Environ. 37, 1586-1597. doi: 10.1111/pce.12258

Furukawa, J., Yamaji, N., Wang, H., Mitani, N., Murata, Y., Sato, K., et al. (2007). An aluminum-activated citrate transporter in barley. Plant Cell Physiol. 48, 1081-1091. doi: 10.1093/pcp/pcm091
Gould, B., McCouch, S., and Geber, M. (2015). De novo transcriptome assembly and identification of gene candidates for rapid evolution of soil $\mathrm{Al}$ tolerance in Anthoxanthum odoratum at the long-term park grass experiment. PLoS ONE 10:e0124424. doi: 10.1371/journal.pone.0124424

Grabherr, M. G., Haas, B. J., Yassour, M., Levin, J. Z., Thompson, D. A., Amit, I., et al. (2011). Full-length transcriptomeassembly from RNA-Seq data without a reference genome. Nat. Biotechnol. 29, 644-652. doi: 10.1038/nbt.1883

Guo, F., Yu, H., Xu, Q., and Deng, X. (2015). Transcriptomic analysis of differentially expressed genes in an orange-pericarp mutant and wild type in pummelo (Citrus grandis). BMC Plant Biol. 15:44. doi: 10.1186/s12870-0150435-3

Horst, W. J., Wang, Y., and Eticha, D. (2010). The role of the root apoplast in aluminium-induced inhibition of root elongation and in aluminium resistance of plants: a review. Ann. Bot. 106, 185-197. doi: 10.1093/aob/mcq053

Hsu, P. H. (1963). Effect of initial pH, phosphate, and silicate on the determination of aluminum with aluminon. Soil Sci. 96, 230-238. doi: 10.1097/00010694196310000-00002

Huang, C. F., Yamaji, N., Mitani, N., Yano, M., Nagamura, Y., and Ma, J. F. (2009). A bacterial-type $\mathrm{ABC}$ transporter is involved in aluminum tolerance in rice. Plant Cell 21, 655-667. doi: 10.1105/tpc.108.064543

Huang, Y. X., Yin, Y. G., Sanuki, A., Fukuda, N., Ezura, H., and Matsukura, C. (2015). Phosphoenolpyruvate carboxykinase (PEPCK) deficiency affects the germination, growth and fruit sugar content in tomato (Solanum lycopersicum L.). Plant Physiol. Biochem. 96, 417-425. doi: 10.1016/j.plaphy.2015.08.021

Huynh, V. B., Repellin, A., Zuily-Fodil, Y., and Pham-Thi, A. T. (2012). Aluminum stress response in rice: effects on membrane lipid composition and expression of lipid biosynthesis genes. Physiol. Plant. 146, 272-284. doi: 10.1111/j.13993054.2012.01622.x

Iuchi, S., Koyama, H., Iuchi, A., Kobayashi, Y., Kitabayashi, S., Kobayashi, Y., et al. (2007). Zinc finger protein STOP1 is critical for proton tolerance in Arabidopsis and coregulates a key gene in aluminum tolerance. Proc. Natl. Acad. Sci. U.S.A. 104, 9900-9905. doi: 10.1073/pnas.0700117104

Jiang, H. X., Tang, N., Zheng, J. G., Li, Y., and Chen, L. S. (2009). Phosphorus alleviates aluminum-induced inhibition of growth and photosynthesis in Citrus grandis seedlings. Physiol. Plant. 137, 298-311. doi: 10.1111/j.1399-3054.2009. 01288.x

Jiang, H. X., Yang, L. T., Qi, Y. P., Lu, Y. B., Huang, Z. R., and Chen, L. S. (2015). Root iTRAQ protein profile analysis of two citrus species differing in aluminum-tolerance in response to long-term aluminum-toxicity. $B M C$ Genomics 16:949. doi: 10.1186/s12864-015-2133-9

Jiang, Y., Chen, R., Dong, J., Xu, Z., and Gao, X. (2012). Analysis of GDSL lipase (GLIP) family genes in rice (Oryza sativa). Plant Omics J. 5, 351-358.

Kim, D., Pertea, G., Trapnell, C., Pimentel, H., Kelley, R., and Salzberg, S. L. (2013). TopHat2: accurate alignment of transcriptomes in the presence of insertions, deletions and gene fusions. Genome Biol. 14:R36. doi: 10.1186/gb-201314-4-r36

Kochian, L. V. (1995). Cellular mechanisms of aluminum toxicity and resistance in plants. Annu. Rev. Plant Physiol. Mol. Biol. 46, 237-260. doi: 10.1146/annurev. pp.46.060195.001321

Kochian, L. V., Piñeros, M. A., Liu, J., and Magalhaes, J. V. (2015). Plant adaptation to acid soils: the molecular basis for crop aluminum resistance. Annu. Rev. Plant Biol. 66, 571-598. doi: 10.1146/annurev-arplant-043014-114822

Kumari, M., Taylor, G. J., and Deyholos, M. K. (2008). Transcriptomic responses to aluminum stress in roots of Arabidopsis thaliana. Mol. Genet. Genomics 279, 339-357. doi: 10.1007/s00438-007-0316-z

Langmead, B., Trapnell, C., Pop, M., and Salzberg, S. L. (2009). Ultrafast and memory-efficient alignment of short DNA sequences to the human genome. Genome Biol. 10:R25. doi: 10.1186/gb-2009-10-3-r25

Larsen, P. B., Cancel, J., Rounds, M., and Ochoa, V. (2007). Arabidopsis ALS1 encodes a root tip and stele localized half type $A B C$ transporter required for root growth in an aluminum toxic environment. Planta 225, 1447-1458. doi: 10.1007/s00425-006-0452-4

Larsen, P. B., Geisler, M. J., Jones, C. A., Williams, K. M., and Cancel, J. D. (2005). ALS3 encodes a phloem-localized ABC transporter-like protein that is required for aluminum tolerance in Arabidopsis. Plant J. 41, 353-363. doi: 10.1111/j.1365-313X.2004.02306.x

Lemire, J., Kumar, P., Mailloux, R., Cossar, K., and Appanna, V. D. (2008). Metabolic adaptation and oxaloacetate homeostasis in P. fluorescens exposed 
to aluminum toxicity. J. Basic Microbiol. 48, 252-259. doi: 10.1002/jobm. 200800007

Li, H., Yang, L. T., Qi, Y. P., Gu, P., Lu, Y. B., and Chen, L. S. (2016). Aluminumtoxicity-induced alterations of leaf proteome in two citrus species differing in aluminum-tolerance. Int. J. Mol. Sci. 17:1180. doi: 10.3390/ijms17071180

Li, J. Y., Liu, J., Dong, D., Jia, X., McCouch, S. R., and Kochian, L. V. (2014). Natural variation underlies alterations in Nramp aluminum transporter (NRAT1) expression and function that play a key role in rice aluminum tolerance. Proc. Natl. Acad. Sci. U.S.A. 111, 6503-6508. doi: 10.1073/pnas.1318975111

Li, P., Deng, W. Q., Li, T. H., Song, B., and Shen, Y. H. (2013). Illumina-based de novo transcriptome sequencing and analysis of Amanita exitialis basidiocarps. Gene 532, 63-71. doi: 10.1016/j.gene.2013.09.014

Li, Y., Han, M. Q., Lin, F., Ten, Y., Lin, J., Zhu, D. H., et al. (2015). Soil chemical properties, 'Guanximiyou' pummelo leaf mineral nutrient status and fruit quality in the southern region of Fujian province, China. J. Soil Sci. Plant Nutr. 15, 615-628. doi: 10.4067/s0718-95162015005000029

Lin, Z., and Myhre, D. L. (1990). Citrus root growth as affected by soil aluminum level under field conditions. Soil Sci. Soc. Am. J. 54, 1340-1344. doi: 10.2136/ sssaj1990.03615995005400050023x

Liu, J., Magalhaes, J. V., Shaff, J., and Kochian, L. V. (2009). Aluminum-activated citrate and malate transporters from the MATE and ALMT families function independently to confer Arabidopsis aluminum tolerance. Plant J. 57, 389-399. doi: 10.1111/j.1365-313X.2008.03696.x

Liu, P. D., Xue, Y. B., Chen, Z. J., Liu, G. D., and Tian, J. (2016). Characterization of purple acid phosphatases involved in extracellular dNTP utilization in Stylosanthes. J. Exp. Bot. 67, 4141-4154. doi: 10.1093/jxb/erw190

Ma, B., Gao, L., Zhang, H., Cui, J., and Shen, Z. (2012). Aluminum-induced oxidative stress and changes in antioxidant defenses in the roots of rice varieties differing in Al tolerance. Plant Cell Rep. 31, 687-696. doi: 10.1007/s00299-011$1187-7$

Ma, J. F., Shen, R. F., Nagao, S., and Tanimoto, E. (2004). Aluminum targets elongating cells by reducing cell wall extensibility in wheat roots. Plant Cell Physiol. 45, 583-589. doi: 10.1093/pcp/pch060

Magalhaes, J. V., Liu, J., Guimarães, C. T., Lana, U. G., Alves, V. M., Wang, Y. H., et al. (2007). A gene in the multidrug and toxic compound extrusion (MATE) family confers aluminum tolerance in sorghum. Nat. Genet. 39, 1156-1161. doi: $10.1038 /$ ng2074

Maron, L. G., Kirst, M., Mao, C., Milner, M. J., Menossi, M., and Kochian, L. V. (2008). Transcriptional profiling of aluminum toxicity and tolerance responses in maize roots. New Phytol. 179, 116-128. doi: 10.1111/j.1469-8137.2008. 02440.x

Mattiello, L., Kirst, M., da Silva, F. R., Jorge, R. A., and Menossi, M. (2010). Transcriptional profile of maize roots under acid soil growth. BMC Plant Biol. 10:196. doi: 10.1186/1471-2229-10-196

Morales, L., Gutiérrez, N., Maya, V., Parra, C., Martínez-Barajas, E., and Coello, P. (2012). Purification and characterization of an alkaline phosphatase induced by phosphorus starvation in common bean (Phaseolus vulgaris L.) roots. J. Mex. Chem. Soc. 56, 80-84.

Mortazavi, A., Williams, B. A., McCue, K., Schaeffer, L., and Wold, B. (2008), Mapping and quantifying mammalian transcriptomes by RNA-Seq. Nat. Methods 5, 621-628. doi: 10.1038/nmeth.1226

Naranjo, M. A., Forment, J., Roldan, M., Serrano, R., and Vicente, O. (2006). Overexpression of Arabidopsis thaliana LTL1, a salt-induced gene encoding a GDSL-motif lipase, increases salt tolerance in yeast and transgenic plants. Plant Cell Environ. 29, 1890-1900. doi: 10.1111/j.1365-3040.2006.01565.x

Okekeogbu, I., Ye, Z., Sangireddy, S. R., Li, H., Bhatti, S., Hui, D., et al. (2014). Effect of aluminum treatment on proteomes of radicles of seeds derived from Altreated tomato plants. Proteomes 2, 169-190. doi: 10.3390/proteomes2020169

Peixoto, P. H. P., Cambraia, J., Sant'Anna, R., Mosquim, P. R., and Moreira, M. A. (1999). Aluminum effects on lipid peroxidation and on the activities of enzymes of oxidative metabolism in sorghum. R. Bras. Fisiol. Veg. 11, 137-143.

Peixoto, P. H. P., Cambraia, J., Sant'Anna, R., Mosquim, P. R., and Moreira, M. A. (2001). Aluminum effects on fatty acid composition and lipid peroxidation of a purified plasma membrane fraction of root apices of two sorghum cultivars. J. Plant Nutr. 24, 1061-1070. doi: 10.1081/PLN-100103803

Pereira, J. F., Zhou, G., Delhaize, E., Richardson, T., Zhou, M., and Ryan, P. R. (2010). Engineering greater aluminium resistance in wheat by over-expressing TaALMT1. Ann. Bot. 106, 205-214. doi: 10.1093/aob/mcq058
Pignocchi, C., Fletcher, J. M., Wilkinson, J. E., Barnes, J. D., and Foyer, C. H. (2003). The function of ascorbate oxidase in tobacco. Plant Physiol. 132, 1631-1641. doi: 10.1104/pp.103.022798

Quartin, V. L., Azinheira, H. G., and Nunes, M. A. (2001). Phosphorus deficiency is responsible for biomass reduction of triticale in nutrient solution with aluminum. J. Plant Nutr. 24, 1901-1911. doi: 10.1081/PLN-100107602

Rengel, Z., and Zhang, W. H. (2003). Role of dynamics of intracellular calcium in aluminium-toxicity syndrome. New Phytol. 159, 295-314. doi: 10.1046/j.14698137.2003.00821.x

Roberts, A., Pimentel, H., Trapnell, C., and Pachter, L. (2011). Identification of novel transcripts in annotated genomes using RNA-Seq. Bioinformatics 27, 2325-2329. doi: 10.1093/bioinformatics/btr355

Roselló, M., Poschenrieder, C., Gunsé, B., Barceló, J., and Llugany, M. (2015). Differential activation of genes related to aluminium tolerance in two contrasting rice cultivars. J. Inorg. Biochem. 152, 160-166. doi: 10.1016/j. jinorgbio.2015.08.021

Sasaki, T., Yamamoto, Y., Ezaki, B., Katsuhara, M., Ahn, S. J., Ryan, P. R., et al. (2004). A wheatgene encoding an aluminum-activated malate transporter. Plant J. 37, 645-653. doi: 10.1111/j.1365-313X.2003.01991.X

Sawaki, Y., Iuchi, S., Kobayashi, Y., Kobayashi, Y., Ikka, T., Sakurai, N., et al. (2009). STOP1 regulates multiple genes that protect arabidopsis from proton and aluminum toxicities. Plant Physiol. 150, 281-294. doi: 10.1104/pp.108.134700

Sivaguru, M., Baluska, F., Volkmann, D., Felle, H. H., and Horst, W. J. (1999). Impacts of aluminum on the cytoskeleton of the maize root apex. Short-term effects on the distal part of the transition zone. Plant Physiol. 119, 1073-1082. doi: $10.1104 /$ pp.119.3.1073

Sivaguru, M., Ezaki, B., He, Z. H., Tong, H., Osawa, H., Baluska, F., et al. (2003). Aluminum-induced gene expression and protein localization of a cell wall-associated receptor kinase in Arabidopsis. Plant Physiol. 132, 2256-2266. doi: 10.1104/pp.103.022129

Sun, L., Liang, C., Chen, Z., Liu, P., Tian, J., Liu, G., et al. (2014). Superior aluminium (Al) tolerance of Stylosanthes is achieved mainly by malate synthesis through an Al-enhanced malic enzyme, SgME1. New Phytol. 202, 209-219. doi: $10.1111 / \mathrm{nph} .12629$

Tabuchi, A., and Matsumoto, H. (2001). Changes in cell-wall properties of wheat (Triticum aestivum) roots during aluminum-induced growth inhibition. Physiol. Plant. 112, 353-358. doi: 10.1034/j.1399-3054.2001.1120308.x

Tamás, L., Budíková, S., Huttová, J., Mistrík, I., Simonovicová, M., and Siroká, B. (2005). Aluminum-induced cell death of barley-root border cells is correlated with peroxidase- and oxalate oxidase-mediated hydrogen peroxide production. Plant Cell Rep. 24, 189-194. doi: 10.1007/s00299-005-0939-7

Teraoka, T., Kaneko, M., Mori, S., and Yoshimura, E. (2002). Aluminum rapidly inhibits cellulose synthesis in roots of barley and wheat seedlings. J. Plant Physiol. 159, 17-23. doi: 10.1078/0176-1617-00678

Trapnell, C., Williams, B. A., Pertea, G., Mortazavi, A., Kwan, G., Van Baren, M. J., et al. (2010). Transcript assembly and quantification by RNA-Seq reveals unannotated transcripts and isoform switching during cell differentiation. Nat. Biotechnol. 28, 511-515. doi: 10.1038/nbt.1621

Wang, L. Q., Yang, L. T., Guo, P., Zhou, X. X., Ye, X., Chen, E. J., et al. (2015). Leaf cDNA-AFLP analysis reveals novel mechanisms for boron-induced alleviation of aluminum-toxicity in Citrus grandis seedlings. Ecotoxicol. Environ. Saf. 120, 349-359. doi: 10.1016/j.ecoenv.2015.06.009

Wang, Z. Q., Xu, X. Y., Gong, Q. Q., Xie, C., Fan, W., Yang, J. L., et al. (2014). Root proteome of rice studied by iTRAQ provides integrated insight into aluminum stress tolerance mechanisms in plants. J. Proteomics 98, 189-205. doi: 10.1016/j.jprot.2013.12.023

$\mathrm{Xu}, \mathrm{R}$. K., and Ji, G. L. (1998). Influence of $\mathrm{pH}$ on disolution of aluminum in acid soils and the distribution of aluminum ion species. Acta Pedol. Sin. 35, 162-171.

Yamaji, N., Huang, C. F., Nagao, S., Yano, M., Sato, Y., Nagamura, Y., et al. (2009). A zinc finger transcription factor ART1 regulates multiple genes implicated in aluminum tolerance in rice. Plant Cell 21, 3339-3349. doi: 10.1105/tpc.109. 070771

Yang, J. L., Zhu, X. F., Peng, Y. X., Zheng, C., Li, G. X., Liu, Y., et al. (2011). Cell wall hemicellulose contributes significantly to aluminum adsorption and root growth in Arabidopsis. Plant Physiol. 155, 1885-1892. doi: 10.1104/pp.111. 172221

Yang, L. T., Jiang, H. X., Qi, Y. P., and Chen, L. S. (2012). Differential expression of genes involved in alternative glycolytic pathways, phosphorus scavenging and 
recycling in response to aluminum and phosphorus interactions in citrus roots. Mol. Biol. Rep. 39, 6353-6366. doi: 10.1007/s11033-012-1457-7

Yang, L. T., Jiang, H. X., Tang, N., and Chen, L. S. (2011). Mechanisms of aluminum-tolerance in two species of Citrus: secretion of organic acid anions and immobilization of aluminum by phosphorus in roots. Plant Sci. 180, 521-530. doi: 10.1016/j.plantsci.2010.11.011

Yang, L. T., Qi, Y. P., Jiang, H. X., and Chen, L. S. (2013). Roles of organic acid anion secretion in aluminium tolerance of higher plants. BioMed. Res. Int. 2013:173682. doi: 10.1155/2013/173682

Yang, Q., Wang, Y., Zhang, J., Shi, W., Qian, C., and Peng, X. (2007). Identification of aluminum-responsive proteins in rice roots by a proteomic approach: cysteine synthase as a key player in Al response. Proteomics 7, 737-749. doi: 10.1002/pmic.200600703

Yin, L., Mano, J., Wang, S., Tsuji, W., and Tanaka, K. (2010). The involvement of lipid peroxide-derived aldehydes in aluminum toxicity of tobacco roots. Plant Physiol. 152, 1406-1417. doi: 10.1104/pp.109.151449

Yokosho, K., Yamaji, N., and Ma, J. F. (2014). Global transcriptome analysis of Alinduced genes in an Al-accumulating species, common buckwheat (Fagopyrum esculentum Moench). Plant Cell Physiol. 55, 2077-2091. doi: 10.1093/pcp/ pcu 135

Yokosho, K., Yamaji, N., Mitani-Ueno, N., Shen, R. F., and Ma, J. F. (2016). An aluminum-inducible IREG gene is required for internal detoxification of aluminum in buckwheat. Plant Cell Physiol. 57, 1169-1178. doi: 10.1093/pcp/ pcw065

You, J., Zhang, H., Liu, N., Gao, L., Kong, L., and Yang, Z. (2011). Transcriptomic responses to aluminum stress in soybean roots. Genome 54, 923-933. doi: $10.1139 / \mathrm{g} 11-060$
Zhang, H., Tan, Z. Q., Hu, L. Y., Wang, S. H., Luo, J. P., and Jones, R. L. (2010). Hydrogen sulfide alleviates aluminum toxicity in germinating wheat seedlings. J. Integr. Plant Biol. 52, 556-567. doi: 10.1111/j.1744-7909.2010. 00946.x

Zhang, M., Deng, X., Yin, L., Qi, L., Wang, X., Wang, S., et al. (2016). Regulation of galactolipid biosynthesis by overexpression of the rice MGD gene contributes to enhanced aluminum tolerance in tobacco. Front. Plant Sci. 7:337. doi: 10.3389/ fpls.2016.00337

Zhou, C. P., Qi, Y. P., You, X., Yang, L. T., Guo, P., Ye, X., et al. (2013). Leaf cDNAAFLP analysis of two citrus species differing in manganese tolerance in response to long-term manganese-toxicity. BMC Genomics 14:621. doi: 10.1186/14712164-14-621

Zhou, X. X., Yang, L. T., Qi, Y. P., Guo, P., and Chen, L. S. (2015). Mechanisms on boron-induced alleviation of aluminum-toxicity in Citrus grandis seedlings at a transcriptional level revealed by cDNA-AFLP analysis. PLoS ONE 10:e0115485. doi: 10.1371/journal.pone.0115485

Conflict of Interest Statement: The authors declare that the research was conducted in the absence of any commercial or financial relationships that could be construed as a potential conflict of interest.

Copyright (c) 2017 Guo, Qi, Yang, Lai, Ye, Yang and Chen. This is an open-access article distributed under the terms of the Creative Commons Attribution License (CC BY). The use, distribution or reproduction in other forums is permitted, provided the original author(s) or licensor are credited and that the original publication in this journal is cited, in accordance with accepted academic practice. No use, distribution or reproduction is permitted which does not comply with these terms. 